\title{
Consistency tests for the extraction of the Boer-Mulders and Sivers functions
}

\author{
E. Christova, ${ }^{1, *}$ E. Leader, ${ }^{2, \dagger}$ and M. Stoilov ${ }^{1, *}$ \\ ${ }^{1}$ Institute for Nuclear Research and Nuclear Energy, Bulgarian Academy of Sciences, \\ Tzarigradsko chaussée 72, 1784 Sofia, Bulgaria \\ ${ }^{2}$ Imperial College London, London SW7 2AZ, United Kingdom
}

(Received 1 June 2017; published 27 March 2018)

\begin{abstract}
At present, the Boer-Mulders (BM) function for a given quark flavor is extracted from data on semiinclusive deep inelastic scattering (SIDIS) using the simplifying assumption that it is proportional to the Sivers function for that flavor. In a recent paper, we suggested that the consistency of this assumption could be tested using information on so-called difference asymmetries i.e. the difference between the asymmetries in the production of particles and their antiparticles. In this paper, using the SIDIS COMPASS deuteron data on the $\left\langle\cos \phi_{h}\right\rangle,\left\langle\cos 2 \phi_{h}\right\rangle$ and Sivers difference asymmetries, we carry out two independent consistency tests of the assumption of proportionality, but here applied to the sum of the valence-quark contributions. We find that such an assumption is compatible with the data. We also show that the proportionality assumptions made in the existing parametrizations of the BM functions are not compatible with our analysis, which suggests that the published results for the Boer-Mulders functions for individual flavors are unreliable. The $\left\langle\cos \phi_{h}\right\rangle$ and $\left\langle\cos 2 \phi_{h}\right\rangle$ asymmetries receive contributions also from the, in principle, calculable Cahn effect. We succeed in extracting the Cahn contributions from experiment (we believe for the first time) and compare with their calculated values, with interesting implications.
\end{abstract}

DOI: 10.1103/PhysRevD.97.056018

\section{INTRODUCTION}

There is a major effort at present to progress beyond a knowledge of collinear parton distribution functions (PDFs) and fragmentation functions (FFs) and to obtain information about the transverse momentum dependent (TMD) versions of these functions. In extracting these distributions from data, a standard parametrization is usually adopted (see for example [1]), which involves various simplifying assumptions. In addition, because of lack of sufficient data, additional relations between different TMD-functions are sometimes assumed. We focus on, and examine, the particular assumption that the BM functions for a particular flavor are proportional to the Sivers functions of the same flavor.

In our recent paper [2], we showed that the difference asymmetries in SIDIS allow the determination of the valence quark TMDs in a model-independent way, without any assumptions about the sea quark or gluon densities. Also, that using the difference asymmetries, one can test many of

\footnotetext{
*echristo@inrne.bas.bg †elliot.leader@cern.ch

*mstoilov@inrne.bas.bg
}

Published by the American Physical Society under the terms of the Creative Commons Attribution 4.0 International license. Further distribution of this work must maintain attribution to the author(s) and the published article's title, journal citation, and DOI. Funded by SCOAP ${ }^{3}$. the basic assumptions in the standard parametrization, such as factorization of the $x_{B}$ and $z_{h}$ dependencies, the Gaussian flavor- and hadron-independent $k_{\perp}$ behavior etc.

In [2], we derived two types of relations-between the $\left\langle\cos \phi_{h}\right\rangle,\left\langle\cos 2 \phi_{h}\right\rangle$ and Sivers asymmetries-that allow tests of the simplifying assumption used in extracting the Boer-Mulders (BM) function i.e. its proportionality to the Sivers function [3,4], an assumption motivated by model calculations [5]. In addition, present analyses make a further assumption concerning the $Q^{2}$ evolution of these functions for a given quark flavor, which, as explained in the next section, is theoretically inconsistent.

Our previously published tests [2] were formulated without taking into account the Cahn effect, which inevitably contributes to these asymmetries. In this paper we show how these tests are modified when the Cahn effect is included.

We then use COMPASS SIDIS measurements of the $\left\langle\cos \phi_{h}\right\rangle,\left\langle\cos 2 \phi_{h}\right\rangle$ and Sivers asymmetries on a deuteron target to test for the consistency of the assumed relation between BM and Sivers functions.

We work with the so-called difference asymmetries of the following general structure. If the asymmetries for $h^{+}$ and $h^{-}$have the form

$$
A^{h^{+}}=\frac{\Delta \sigma^{h^{+}}}{\sigma^{h^{+}}} \quad A^{h^{-}}=\frac{\Delta \sigma^{h^{-}}}{\sigma^{h^{-}}}
$$


where $\sigma^{h^{+}, h^{-}}$and $\Delta \sigma^{h^{+}, h^{-}}$are the unpolarized and polarized cross sections, respectively, then

$$
A^{h^{+}-h^{-}} \equiv \frac{\Delta \sigma^{h^{+}}-\Delta \sigma^{h^{-}}}{\sigma^{h^{+}}-\sigma^{h^{-}}} .
$$

The difference asymmetries are expressed in terms of the usual asymmetries $A^{h^{+}, h^{-}}$and the ratio of the corresponding multiplicities [6],

$$
A^{h^{+}-h^{-}}=\frac{1}{1-r}\left(A^{h^{+}}-r A^{h^{-}}\right),
$$

where $r$ is the ratio of unpolarized SIDIS cross sections for production of $h^{-}$and $h^{+}: r=\sigma^{h^{-}} / \sigma^{h^{+}}$.

As shown in Ref. [2], the advantage of using the difference asymmetries is that, based only on charge conjugation (C) and isospin (SU(2)) invariance of the strong interactions, they are expressed purely in terms of the best known valence-quark distributions and fragmentation functions; sea-quark and gluon distributions do not enter. For a deuteron target there is the additional simplification that, independently of the final hadron, only the sum of the valence-quark distributions enters.

The paper is organized as follows: the notation and conventions for the various TMD functions and the used experimental asymmetries are explained in Secs. II and III; in Sec. IV, we formulate the two tests for the assumed relation between the BM and Sivers functions. They are based on the $\left\langle\cos \phi_{h}\right\rangle$ and $\left\langle\cos 2 \phi_{h}\right\rangle$ azimuthal asymmetries of the final hadrons in unpolarized SIDIS, and the Sivers asymmetry for unpolarized leptons on transversely polarized nucleons. Because the above two unpolarized asymmetries receive contributions from both the BM and Cahn effects, we are able also to extract information about the Cahn effect; in Sec. V, we apply these tests using the COMPASS SIDIS data on deuterons.

\section{PARAMETRIZATION OF THE TMD DISTRIBUTIONS}

\section{A. The polarized parton distribution functions}

Conventionally, a typical spin-dependent TMD density $\Delta f\left(k_{\perp}, x_{B}, Q^{2}\right)$ has been parametrized following several simplifying assumptions:

(1) The transverse-momentum dependence on $k_{\perp}$ is factorized from the $x_{B}$ dependence.

(2) The $k_{\perp}$ dependence is flavor and hadron independent, and usually assumed to be a Gaussian.

We adopt these two simplifications.

(3) An additional simplifying assumption is that TMD functions are proportional to the related collinear parton distribution functions (PDFs) and fragmentation functions (FFs). The $Q^{2}$-evolution is usually assumed to be given via the collinear PDFs and FFs, i.e. making the ansatz:

$$
\begin{aligned}
& \Delta f_{q}\left(x_{B}, Q^{2}\right)=2 \mathcal{N}_{q}\left(x_{B}\right) q\left(x_{B}, Q^{2}\right) \\
& \Delta f_{\bar{q}}\left(x_{B}, Q^{2}\right)=2 \mathcal{N}_{\bar{q}}\left(x_{B}\right) \bar{q}\left(x_{B}, Q^{2}\right)
\end{aligned}
$$

This is, however, physically unacceptable because it leads to gluons contributing to the evolution of nonsinglet combinations of quark densities.

Since we deal here only with valence quark densities we replace this simplification by an ansatz for the valencequark densities. Hence we take the $Q^{2}$ evolution to be controlled via:

$$
\begin{aligned}
\Delta f_{q_{V}}\left(x_{B}, Q^{2}\right) & =2 \mathcal{N}_{q_{V}}\left(x_{B}\right) q_{V}\left(x_{B}, Q^{2}\right), \\
q_{V} & =u_{V}, d_{V}
\end{aligned}
$$

Note, however, that we do not think this difference in approximating the evolution is important when assessing the impact of our tests on the published BM data.

In this paper we consider only the difference asymmetries on a deuteron target. As mentioned earlier, in these asymmetries only one combination of parton density enters - the sum of the valence-quark TMD functions:

$\Delta f_{Q_{V}}\left(x_{B}, k_{\perp}, Q^{2}\right) \equiv \Delta f_{u_{V}}\left(x_{B}, k_{\perp}, Q^{2}\right)+\Delta f_{d_{V}}\left(x_{B}, k_{\perp}, Q^{2}\right)$

Below we present the parametrizations of the valencequark $Q_{V}$ unpolarized, $\mathrm{BM}$ and Sivers distributions and the Collins FFs following the above simplifying anzatz. We work in the approximation $\mathcal{O}\left(k_{\perp} / Q\right)$, neglecting terms of the order $\mathcal{O}\left(k_{\perp}^{2} / Q^{2}\right)$.

\section{B. The unpolarized TMD parton distributions and fragmentation functions}

The unpolarized TMD PDFs and FFs are parametrized proportional to the corresponding collinear functions times a Gaussian-type, flavor- and hadron-independent $k_{\perp}^{2} / p_{\perp}^{2}$ dependence [7]. In accordance with this for the valencequark unpolarized TMD PDFs $f_{Q_{V} / p}\left(x_{B}, k_{\perp}^{2}, Q^{2}\right)$ and TMD FFs $D_{h / q_{V}}\left(z_{h}, p_{\perp}^{2}, Q^{2}\right)$ we adopt the parametrizations [8]:

$$
f_{Q_{V} / p}\left(x_{B}, k_{\perp}^{2}, Q^{2}\right)=Q_{V}\left(x_{B}, Q^{2}\right) \frac{e^{-k_{\perp}^{2} /\left\langle k_{\perp}^{2}\right\rangle}}{\pi\left\langle k_{\perp}^{2}\right\rangle}
$$

and

$$
D_{h / q_{V}}\left(z_{h}, p_{\perp}^{2}, Q^{2}\right)=D_{q_{V}}^{h}\left(z_{h}, Q^{2}\right) \frac{e^{-p_{\perp}^{2} /\left\langle p_{\perp}^{2}\right\rangle}}{\pi\left\langle p_{\perp}^{2}\right\rangle},
$$

where $Q_{V}\left(x_{B}, Q^{2}\right)$ is the sum of the collinear valence-quark PDFs:

$$
Q_{V}\left(x_{B}, Q^{2}\right)=u_{V}\left(x_{B}, Q^{2}\right)+d_{V}\left(x_{B}, Q^{2}\right)
$$

and $D_{q_{V}}^{h}\left(z_{h}, Q^{2}\right)$ are the valence-quark collinear FFs: 


$$
D_{q_{V}}^{h}\left(z_{h}, Q^{2}\right)=D_{q}^{h}\left(z_{h}, Q^{2}\right)-D_{\bar{q}}^{h}\left(z_{h}, Q^{2}\right),
$$

$\left\langle k_{\perp}^{2}\right\rangle$ and $\left\langle p_{\perp}^{2}\right\rangle$ are parameters extracted from study of the multiplicities in unpolarized SIDIS.

The parameters $\left\langle k_{\perp}^{2}\right\rangle$ (and $\left\langle p_{\perp}^{2}\right\rangle$ are basic as they enter in the normalization functions in all TMD asymmetries. At present the experimentally obtained values are controversial:

(1) $\left\langle k_{\perp}^{2}\right\rangle \approx 0.25 \mathrm{GeV}^{2}$ and $\left\langle p_{\perp}^{2}\right\rangle \approx 0.20 \mathrm{GeV}^{2}$ [9], extracted from the old EMC [10] and FNAL [11] SIDIS data

(2) $\left\langle k_{\perp}^{2}\right\rangle=0.18 \mathrm{GeV}^{2}$ and $\left\langle p_{\perp}^{2}\right\rangle=0.20 \mathrm{GeV}^{2}$ [12], derived from the $P_{T}$-spectrum of HERMES data and confirmed by Monte Carlo calculations. The extraction of the BM functions in [4] utilized these values.

An analysis [7] of the more recent available data on multiplicities from HERMES [13] and COMPASS [14] separately, gives quite different values:

(3) $\left\langle k_{\perp}^{2}\right\rangle=0.57 \pm 0.08 \mathrm{GeV}^{2}$ and $\left\langle p_{\perp}^{2}\right\rangle=0.12 \pm$ $0.01 \mathrm{GeV}^{2}$, extracted from HERMES data

(4) $\left\langle k_{\perp}^{2}\right\rangle=0.61 \pm 0.20 \mathrm{GeV}^{2} \quad$ and $\quad\left\langle p_{\perp}^{2}\right\rangle=0.19 \pm$ $0.02 \mathrm{GeV}^{2}$, extracted from COMPASS data.

These values are obtained using a kinematical cut on $z_{h}<0.6$ and they change slightly on placing the cut at $z_{h}<0.7$.

Further we shall be able to comment on this controversial situation, since the Cahn effect, which contributes to the asymmetries which we study and extract from data, is calculable, and depends sensitively on $\left\langle k_{\perp}^{2}\right\rangle$ and $\left\langle p_{\perp}^{2}\right\rangle$.

\section{The BM and Sivers distributions}

The Sivers function describes the correlation between the spin of the nucleon $\mathbf{S}$, its momentum $\mathbf{P}$, and the momentum of the quark $\mathbf{k}_{\perp}$, via a term proportional to $\mathbf{S} \cdot\left(\mathbf{k}_{\perp} \times \mathbf{P}\right)$ [15], while the $\mathrm{BM}$ function describes the correlation between the spin of the quark $\mathbf{s}_{q}$ and the momentum of the quark $\mathbf{k}_{\perp}$, via a term proportional to $\mathbf{s}_{\mathbf{q}} \cdot\left(\mathbf{k}_{\perp} \times \mathbf{P}\right)$ [16].

The $k_{\perp}, x_{B}$ dependence of the valence-quark BM and Sivers distribution functions $\Delta f_{J}^{Q_{V}}\left(x_{B}, k_{\perp}, Q^{2}\right),(J=\mathrm{BM}$, Sivers), is assumed to factorize $[1,4]$ in the form

$$
\begin{aligned}
\Delta f_{J}^{Q_{V}}\left(x_{B}, k_{\perp}, Q^{2}\right) & =\Delta f_{J}^{Q_{V}}\left(x_{B}, Q^{2}\right) \sqrt{2 e} \frac{k_{\perp}}{M_{J}} \frac{e^{-k_{\perp}^{2} /\left\langle k_{\perp}^{2}\right\rangle_{J}}}{\pi\left\langle k_{\perp}^{2}\right\rangle_{J}}, \\
J & =\mathrm{BM}, \text { Sivers }
\end{aligned}
$$

with

$$
\Delta f_{J}^{Q_{V}}\left(x_{B}, Q^{2}\right)=2 \mathcal{N}_{J}^{Q_{V}}\left(x_{B}\right) Q_{V}\left(x_{B}, Q^{2}\right)
$$

Here, the $\mathcal{N}_{J}^{Q_{V}}\left(x_{B}\right)$ are unknown functions, and $M_{J}$, or equivalently $\left\langle k_{\perp}^{2}\right\rangle_{J}$, where

$$
\left\langle k_{\perp}^{2}\right\rangle_{J}=\frac{\left\langle k_{\perp}^{2}\right\rangle M_{J}^{2}}{\left\langle k_{\perp}^{2}\right\rangle+M_{J}^{2}},
$$

are unknown parameters. As mentioned earlier, $\left\langle k_{\perp}^{2}\right\rangle$ is supposed to be known from multiplicities in unpolarized SIDIS.

\section{The Collins fragmentation functions}

The Collins fragmentation functions (FFs) $\Delta^{N} D_{h / q \uparrow}\left(z, p_{\perp}\right)$ describe phenomenologically the spindependent part of the fragmentation functions of transversely polarized quarks, with transverse spin $\mathbf{s}_{q}$ and 3-momentum $\mathbf{p}_{q}$, into hadrons $h$ with momentum $\mathbf{p}_{\perp}$, transverse to the direction of the initial quark [17]:

$$
\begin{aligned}
D_{h / q, s}\left(z_{h}, p_{\perp}\right)= & D_{h / q}\left(z_{h}, p_{\perp}\right) \\
& +\frac{1}{2} \Delta^{N} D_{h / q \uparrow}\left(z_{h}, p_{\perp}\right) \hat{\mathbf{s}}_{q} \cdot\left(\hat{\mathbf{p}}_{q} \times \hat{\mathbf{p}}_{\perp}\right) .
\end{aligned}
$$

It relates the transverse momentum of the produced hadron to the transverse spin of the quark and leads to nonuniform azimuthal distribution of final hadrons around the initial quark direction.

The valence-quark Collins functions $\Delta^{N} D_{h / u_{V} \uparrow}\left(z_{h}, p_{\perp}\right.$, $Q^{2}$ ) are parametrized [2] proportional to the corresponding unpolarized valence-quark collinear fragmentation functions $D_{u_{V}}^{h}\left(z_{h}, Q^{2}\right)$ :

$$
\begin{aligned}
\Delta^{N} D_{h / u_{V} \uparrow}\left(z_{h}, p_{\perp}, Q^{2}\right)= & \Delta^{N} D_{h / u_{V} \uparrow}\left(z_{h}, Q^{2}\right) \\
& \times \sqrt{2 e} \frac{p_{\perp}}{M_{C}} \frac{e^{-p_{\perp}^{2} /\left\langle p_{\perp}^{2}\right\rangle_{C}}}{\pi\left\langle p_{\perp}^{2}\right\rangle_{C}}, \\
h= & \pi^{+}, K^{+}, h^{+}
\end{aligned}
$$

where

$$
\Delta^{N} D_{h / u_{V} \uparrow}\left(z_{h}, Q^{2}\right)=2 \mathcal{N}_{C}^{h / u_{V}}\left(z_{h}\right) D_{u_{V}}^{h}\left(z_{h}, Q^{2}\right) .
$$

The unknown quantities are $\mathcal{N}_{C}^{h / u_{V}}\left(z_{h}\right)$ and $M_{C}$ (often $M_{C}$ is denoted by $M$ [18] or $\left.M_{h}[1,19]\right)$, or equivalently $\left\langle p_{\perp}^{2}\right\rangle_{C}$ :

$$
\left\langle p_{\perp}^{2}\right\rangle_{C}=\frac{\left\langle p_{\perp}^{2}\right\rangle M_{C}^{2}}{\left\langle p_{\perp}^{2}\right\rangle+M_{C}^{2}}
$$

which characterizes the $p_{\perp}$ dependence. As mentioned earlier, $\left\langle p_{\perp}^{2}\right\rangle$ is known from multiplicities in unpolarized SIDIS.

\section{THE UNPOLARIZED AZIMUTHAL AND SIVERS ASYMMETRIES}

The general expression for the difference cross section in SIDIS, for unpolarized leptons on transversely polarized 
nucleons, with polarization $S_{T}, l+N^{\uparrow} \rightarrow l+h+X$, in the kinematic region $P_{T} \simeq k_{\perp} \ll Q$, is given in terms of the unpolarized $F_{U U}^{h-\bar{h}}, F_{U U}^{\cos \phi_{h}, h-\bar{h}}, F_{U U}^{\cos 2 \phi_{h}, h-\bar{h}}$ and transversely polarized $F_{U T}^{\sin \left(\phi_{S}-\phi_{h}\right), h-\bar{h}}$ structure functions, by [1]:

$$
\begin{aligned}
& \frac{d \sigma_{N}^{h-\bar{h}}}{d x_{B} d Q^{2} d z_{h} d^{2} \mathbf{P}_{T} d \phi_{S}} \\
& =\frac{2 \pi \alpha_{e m}^{2}}{Q^{4}}\left\{\left[1+(1-y)^{2}\right] F_{U U}^{h-\bar{h}}+2(1-y) \cos 2 \phi_{h} F_{U U}^{\cos 2 \phi_{h}, h-\bar{h}}\right. \\
& +2(2-y) \sqrt{1-y} \cos \phi_{h} F_{U U}^{\cos \phi_{h}, h-\bar{h}} \\
& \left.+S_{T}\left[\left[1+(1-y)^{2}\right] \sin \left(\phi_{S}-\phi_{h}\right) F_{U T}^{\sin \left(\phi_{S}-\phi_{h}\right), h-\bar{h}}+\cdots\right]\right\}
\end{aligned}
$$

Here we have kept only the terms relevant to the considerations in this paper: $F_{U U}^{\cos 2 \phi_{h}, h-\bar{h}}$ and $F_{U U}^{\cos \phi_{h}, h-\bar{h}}$ get contributions from both the BM functions and the purely kinematic Cahn effect; $F_{U T}^{\sin \left(\phi_{S}-\phi_{h}\right), h-\bar{h}}$ gets a contribution from the Sivers function; $F_{U U}^{h-\bar{h}}$ determines the unpolarized cross section without $\phi_{h}$ dependence. They involve convolutions of the corresponding valence-quark TMD parton densities and FFs [2,8].

Here $P_{T}$ is the transverse momentum of the final hadron in the $\gamma^{*}$-nucleon c.m. frame, and $z_{h}, Q^{2}$ and $y$ are the usual measurable SIDIS quantities:

$$
\begin{gathered}
z_{h}=\frac{\left(P \cdot P_{h}\right)}{(P \cdot q)}, \quad Q^{2}=-q^{2}, \\
q=l-l^{\prime}, \quad y=\frac{(P \cdot q)}{(P \cdot l)}
\end{gathered}
$$

with $l$ and $l^{\prime}, P$ and $P_{h}$ the 4-momenta of the initial and final leptons, and initial and final hadrons. Note that

$$
Q^{2}=2 M E x_{B} y
$$

where $M$ is the target mass (in this paper the deuteron mass) and $E$ the lepton laboratory energy. Throughout the paper we follow the notation and kinematics of Ref. [1].

In current analyses [3,4], in extracting the BM function, an additional simplifying assumption is made, namely, the BM function is taken proportional to its chiral-even partner-the Sivers function. Clearly the resulting BM function depends critically on the validity of this assumption. Our fundamental aim is to check this key assumption using only measurable quantities - the difference asymmetries - and without requiring any knowledge about the TMD functions.

The difference azimuthal $\cos \phi_{h}, \cos 2 \phi_{h}$ and $\sin \left(\phi_{S}-\phi_{h}\right)$, Sivers, asymmetries that single out these terms are:

$$
\begin{gathered}
A_{U U}^{\cos \phi_{h}, h-\bar{h}}=\frac{\int d \phi_{h} \cos \phi_{h} d \sigma^{h-\bar{h}}}{\int d \phi_{h} d \sigma^{h-\bar{h}}} \\
A_{U U}^{\cos 2 \phi_{h}, h-\bar{h}}=\frac{\int d \phi_{h} \cos 2 \phi_{h} d \sigma^{h-\bar{h}}}{\int d \phi_{h} d \sigma^{h-\bar{h}}} \\
A_{U T}^{\mathrm{Siv}, h-\bar{h}}=\frac{1}{S_{T}} \frac{\int d \phi_{h} d \phi_{S} \sin \left(\phi_{S}-\phi_{h}\right)\left(d \sigma^{\uparrow}-d \sigma^{\downarrow}\right)^{h-\bar{h}}}{\int d \phi_{h} d \phi_{S}\left(d \sigma^{\uparrow}+d \sigma^{\downarrow}\right)^{h-\bar{h}}}
\end{gathered}
$$

The corresponding $x_{B}$-dependent asymmetries, integrated over $P_{T}^{2}, z_{h}$ and $Q^{2}$, that we shall work with are

$$
\begin{aligned}
& A_{U U}^{\cos \phi_{h}, h-\bar{h}}\left(x_{B}\right) \\
& =\frac{\int d Q^{2} d z_{h} d P_{T}^{2}\left[(2-y) \sqrt{1-y} / Q^{4}\right] F_{U U}^{\cos \phi_{h}, h-\bar{h}}}{\int d Q^{2} d z_{h} d P_{T}^{2}\left[\left[1+(1-y)^{2}\right] / Q^{4}\right] F_{U U}^{h-\bar{h}}} \\
& A_{U U}^{\cos 2 \phi_{h}, h-\bar{h}}\left(x_{B}\right) \\
& =\frac{\int d Q^{2} d z_{h} d P_{T}^{2}\left[(1-y) / Q^{4}\right] F_{U U}^{\cos 2 \phi_{h}, h-\bar{h}}}{\int d Q^{2} d z_{h} d P_{T}^{2}\left[\left[1+(1-y)^{2}\right] / Q^{4}\right] F_{U U}^{h-\bar{h}}} \\
& A_{U T}^{\mathrm{Siv}, h-\bar{h}}\left(x_{B}\right) \\
& =\frac{1}{S_{T}} \frac{\int d Q^{2} d z_{h} d P_{T}^{2}\left[\left[1+(1-y)^{2}\right] / Q^{4}\right] F_{U T}^{\sin \left(\phi_{s}-\phi_{h}\right), h-\bar{h}}}{\int d Q^{2} d z_{h} d P_{T}^{2}\left[\left[1+(1-y)^{2}\right] / Q^{4}\right] F_{U U}^{h-\bar{h}}} \\
& \text { IV. TESTS FOR THE RELATION BETWEEN } \\
& \text { THE BM AND SIVERS FUNCTIONS } \\
& \text { ON A DEUTERON TARGET }
\end{aligned}
$$

In the difference asymmetries on deuterium, only the sum of the valence-quarks $Q_{V}=u_{V}+d_{V}$ enters for any final hadron $h$. Therefore, in contrast to the currently used assumption of proportionality between BM and Sivers functions for each quark and antiquark flavor, we assume the simpler relation:

$$
\begin{aligned}
\Delta f_{\mathrm{BM}}^{Q_{V}}\left(x, k_{\perp}, Q^{2}\right) & =\lambda_{Q_{V}} \Delta f_{\mathrm{Siv}}^{Q_{V}}\left(x, k_{\perp}, Q^{2}\right), \\
Q_{V} & =u_{V}+d_{V}
\end{aligned}
$$

where $\lambda_{Q_{V}}$ is a constant. Using the parametrizations (11), Eq. (27) implies that the $k_{\perp}$ dependencies in BM and Sivers functions are the same, while the $x_{B}$ dependencies are proportional:

$M_{\mathrm{BM}}=M_{S}, \quad\left\langle k_{\perp}^{2}\right\rangle_{\mathrm{BM}}=\left\langle k_{\perp}^{2}\right\rangle_{S}, \quad \mathcal{N}_{\mathrm{BM}}^{Q_{V}}\left(x_{B}\right)=\lambda_{Q_{V}} \mathcal{N}_{\mathrm{Siv}}^{Q_{V}}\left(x_{B}\right)$. 
The $\left\langle\cos \phi_{h}\right\rangle$ and $\left\langle\cos 2 \phi_{h}\right\rangle$ azimuthal asymmetries in unpolarized SIDIS receive contributions from both the BM function and the purely kinematic Cahn effect. The connection (27) between the BM and Sivers functions leads to relations between the $\mathrm{BM}$ induced contributions in $\left\langle\cos \phi_{h}\right\rangle$ or $\left\langle\cos 2 \phi_{h}\right\rangle$ and the Sivers asymmetries. Here we present the resulting relations between the $x_{B}$-dependent $\left\langle\cos \phi_{h}\right\rangle$ or $\left\langle\cos 2 \phi_{h}\right\rangle$ and Sivers asymmetries.

These relations are particularly simple and predictive if the bins in $x_{B}$ are small enough, so as to neglect the $Q^{2}$-evolution of the collinear functions inside the bins.
A. Tests based on the asymmetry $A_{U U}^{\cos \phi_{h}}$

Here we present the relation between the $x_{B}$-dependent $\cos \phi_{h}$ and Sivers asymmetries on a deuteron target, when the $Q^{2}$-evolution of the collinear parton densities and fragmentation functions can be neglected inside the considered $x_{B}$-bin. The standard parametrizations (7), (8) and (11), (12) are used.

(1) The asymmetry $A_{U U}^{\cos \phi_{h}}$ has two twist-3 contributions of $1 / Q$-order from the $\mathrm{BM}$ function and from the Cahn effect. For the $x_{B}$-dependent difference asymmetry on a deuteron $A_{U U, d}^{\cos \phi_{h}, h-\bar{h}}\left(x_{B}\right)$, we have (see Appendix A)

$$
A_{U U, d}^{\cos \phi_{h}, h-\bar{h}}\left(x_{B}\right)=\Phi\left(x_{B}\right)\left\{C_{\mathrm{Cahn}}^{h}+2 \mathcal{N}_{\mathrm{BM}}^{Q_{V}}\left(x_{B}\right) C_{\mathrm{BM}}^{h}\right\}, \quad h=\pi^{+}, K^{+}, h^{+}
$$

Here $C_{\text {Cahn }}^{h}$ and $C_{\mathrm{BM}}^{h}$ are constants, given by:

$$
\begin{gathered}
C_{\mathrm{Cahn}}^{h}=-\left\langle k_{\perp}^{2}\right\rangle \frac{\int d z_{h} z_{h}\left[D_{q_{V}}^{h}\left(z_{h}\right)\right] / \sqrt{\left\langle P_{T}^{2}\right\rangle}}{\int d z_{h}\left[D_{q_{V}}^{h}\left(z_{h}\right)\right]} \\
C_{\mathrm{BM}}^{h}=\frac{e\left\langle k_{\perp}^{2}\right\rangle_{\mathrm{BM}}^{2}\left\langle p_{\perp}^{2}\right\rangle_{C}^{2}}{2 M_{\mathrm{BM}} M_{C}\left\langle k_{\perp}^{2}\right\rangle\left\langle p_{\perp}^{2}\right\rangle} \frac{\int d z_{h}\left[z_{h}^{2}\left\langle k_{\perp}^{2}\right\rangle_{\mathrm{BM}}+2\left\langle p_{\perp}^{2}\right\rangle_{C}\right]\left[\Delta^{N} D_{q_{V} \uparrow}^{h}\left(z_{h}\right)\right] /\left\langle P_{T}^{2}\right\rangle_{\mathrm{BM}}^{3 / 2}}{\int d z_{h}\left[D_{u_{V}}^{h}\left(z_{h}\right)\right]} \\
\left\langle P_{T}^{2}\right\rangle=\left\langle p_{\perp}^{2}\right\rangle+z_{h}^{2}\left\langle k_{\perp}^{2}\right\rangle, \quad\left\langle P_{T}^{2}\right\rangle_{\mathrm{BM}}=\left\langle p_{\perp}^{2}\right\rangle_{C}+z_{h}^{2}\left\langle k_{\perp}^{2}\right\rangle_{\mathrm{BM}} .
\end{gathered}
$$

The function $\Phi\left(x_{B}\right)$ is completely fixed by kinematics, the same for all final hadrons:

$$
\Phi\left(x_{B}\right)=\frac{\sqrt{\pi}(2-\bar{y}) \sqrt{1-\bar{y}}}{\langle Q\rangle\left[1+(1-\bar{y})^{2}\right]},
$$

where

$$
\bar{y}=\frac{\langle Q\rangle^{2}}{2 M_{d} E x_{B}},
$$

$\langle Q\rangle^{2}$ is some mean value of $Q^{2}$ for each $x_{B}$-bin (see Appendix A), $M_{d}$ is the mass of the deuterium target.

The notation $\left[D_{q_{V}}^{h}\right]$ is shorthand for the following:

$$
\left[D_{q_{V}}^{h}\left(z_{h}, Q^{2}\right)\right]=D_{u_{V}}^{h}, \quad \text { for } h=\pi^{+}, K^{+}
$$

and

$\left[D_{q_{V}}^{h^{+}}\left(z_{h}, Q^{2}\right)\right]=e_{u}^{2} D_{u_{V}}^{h^{+}}+e_{d}^{2} D_{d_{V}}^{h^{+}}$,

for unidentified charged hadrons $h=h^{+}$.

Analogously for $\left[\Delta^{N} D_{q_{V} \uparrow}^{h}\left(z_{h}\right)\right]$ we have:

$$
\left[\Delta^{N} D_{q_{V} \uparrow}^{h}\left(z_{h}, Q^{2}\right)\right]=\Delta^{N} D_{u_{V} \uparrow}^{h}\left(z_{h}\right), \quad h=\pi^{+}, K^{+}
$$

$$
\left[\Delta^{N} D_{q_{V} \uparrow}^{h^{+}}\left(z_{h}, Q^{2}\right)\right]=e_{u}^{2} \Delta^{N} D_{u_{V} \uparrow}^{h^{+}}+e_{d}^{2} \Delta^{N} D_{d_{V} \uparrow}^{h^{+}} .
$$

(2) Following the same path, for the $x_{B}$-dependent Sivers difference asymmetry on a deuteron $A_{U T, d}^{\mathrm{Siv}, h-\bar{h}}\left(x_{B}\right)$, when the $Q^{2}$ dependence in $Q_{V}\left(x_{B}, Q^{2}\right)$ and in the valence-quark FFs $D_{u_{V}}^{h}\left(z_{h}, Q^{2}\right)$ can be neglected, we obtain [2]:

$$
\begin{aligned}
A_{U T, d}^{\mathrm{Siv}, h-\bar{h}}\left(x_{B}\right) & =\frac{\sqrt{e \pi}}{2 \sqrt{2}} A_{\mathrm{Siv}} C_{\mathrm{Siv}}^{h} \mathcal{N}_{\mathrm{Siv}}^{Q_{V}}\left(x_{B}\right), \\
h= & \pi^{+}, K^{+}, h^{+} \\
A_{\mathrm{Siv}} & =\frac{\left\langle k_{\perp}^{2}\right\rangle_{S}^{2}}{M_{S}\left\langle k_{\perp}^{2}\right\rangle}, \\
C_{\mathrm{Siv}}^{h} & =\frac{\int d z_{h} z_{h}\left[D_{u_{V}}^{h}\right] / \sqrt{\left\langle P_{T}^{2}\right\rangle_{S}}}{\int d z_{h}\left[D_{u_{V}}^{h}\right]} \\
\left\langle P_{T}^{2}\right\rangle_{S} & =\left\langle p_{\perp}^{2}\right\rangle+z_{h}^{2}\left\langle k_{\perp}^{2}\right\rangle_{S}
\end{aligned}
$$


Note that both in $A_{U U, d}^{\cos \phi_{h}, h-\bar{h}}\left(x_{B}\right)$ and in $A_{\mathrm{Siv}, d}^{h-\bar{h}}\left(x_{B}\right)$ (a) there is no sum over quark flavor and (b) the parton density $Q_{V}$ cancels out, being the same in the numerator and denominator.

(3) If the BM distribution is related to Sivers distribution by relations (27) we have:

$$
\begin{aligned}
\mathcal{N}_{\mathrm{BM}}^{Q_{V}}\left(x_{B}\right) & =\lambda_{Q_{V}} \mathcal{N}_{\mathrm{Siv}}^{Q_{V}}\left(x_{B}\right) \\
& =\lambda_{Q_{V}} \frac{2 \sqrt{2}}{\sqrt{e \pi}} \frac{1}{A_{\mathrm{Siv}} C_{\mathrm{Siv}}^{h}} A_{U T, d}^{\mathrm{Siv}, h-\bar{h}}\left(x_{B}\right),
\end{aligned}
$$

which expresses the unknown $x_{B}$ dependence of the BM-distribution in terms of the measurable $x_{B^{-}}$ dependent Sivers asymmetry. The assumed relation (27) between the BM and Sivers functions then leads to the following relation between the $x_{B}$-dependent azimuthal $\cos \phi_{h}$-asymmetry $A_{U U}^{\cos \phi_{h}} \equiv\left\langle\cos \phi_{h}\right\rangle$ and the Sivers asymmetry on a deuteron target:

$$
\begin{gathered}
A_{U U, d}^{\cos \phi_{h}, h-\bar{h}}\left(x_{B}\right)-C_{\mathrm{BM}}^{h} \Phi\left(x_{B}\right) A_{U T, d}^{\mathrm{Siv}, h-\bar{h}}\left(x_{B}\right) \\
=C_{\mathrm{Cahn}}^{h} \Phi\left(x_{B}\right), \quad h=\pi^{+}, K^{+}, h^{+} .
\end{gathered}
$$

Here the function $\Phi\left(x_{B}\right)$ and the constant $C_{\text {Cahn }}^{h}$ are given by (33) and (30), respectively; the constant $C_{\overparen{B M}}^{h}$, induced by the BM function, is obtained from the expression for the coefficient $C_{\mathrm{BM}}^{h}$, Eq. (31), by making the replacements $M_{\mathrm{BM}} \rightarrow M_{S}$ and $\left\langle k_{\perp}^{2}\right\rangle_{\mathrm{BM}} \rightarrow$ $\left\langle k_{\perp}^{2}\right\rangle_{S}$, yielding:

$$
C_{\overparen{\mathrm{BM}}}^{h}=2 \lambda_{Q_{V}} \sqrt{\frac{2 e}{\pi}} \frac{\left\langle p_{\perp}^{2}\right\rangle_{C}^{2}}{M_{C}\left\langle p_{\perp}^{2}\right\rangle} \frac{\int_{0.2}^{1} d z_{h}\left[z_{h}^{2}\left\langle k_{\perp}^{2}\right\rangle_{S}+2\left\langle p_{\perp}^{2}\right\rangle_{C}\right]\left[\Delta^{N} D_{q_{V} \uparrow}^{h}\left(z_{h}\right)\right] /\left\langle P_{T}^{2}\right\rangle \frac{3 / 2}{\mathrm{BM}}}{\int_{0.2}^{1} d z_{h} z_{h}\left[D_{q_{V}}^{h}\left(z_{h}\right)\right] / \sqrt{\left\langle P_{T}^{2}\right\rangle_{S}}}
$$

where

$$
\left\langle P_{T}^{2}\right\rangle_{\mathrm{BM}}=\left\langle p_{\perp}^{2}\right\rangle_{C}+z_{h}^{2}\left\langle k_{\perp}^{2}\right\rangle_{S} .
$$

There are two important consequences of Eq. (42), which we shall use further:

(1) It represents a direct and simple test of the relation (27) between the BM and Sivers TMD-functions, in which only measurable quantities enter, and no knowledge about the TMD functions is required.

(2) The different $x_{B}$ dependences of the Cahn and BM contributions, allow us to disentangle the Cahn contribution from the $\mathrm{BM}$ one in our fits to the experimental data.

\section{B. Tests based on the asymmetry $A_{U U}^{\cos 2 \phi_{h}}$}

(1) The asymmetry $A_{U U}^{\cos 2 \phi_{h}}$ has two contributions: the leading twist-2 contribution from $\mathrm{BM}$ function and the twist-4 contribution of $1 / Q^{2}$-order from the Cahn effect.

Following the same path as in obtaining Eq. (29) (details are given in Appendix B), we obtain the $x_{B^{-}}$ dependent difference asymmetry on a deuteron, $A_{U U, d}^{\cos 2 \phi_{h}, h-\bar{h}}\left(x_{B}\right)$. The only difference is that the integration from the convolution in $\mathbf{k}_{\perp}$, in the contribution from the Cahn effect, cannot be carried out analytically and it remains in the final expressions-these are the integrals over $\phi$ and $k_{\perp}$ in Eq. (48). Here we give only the final expression.

For the $x_{B}$-dependent difference asymmetry on a deuteron $A_{U U, d}^{\cos 2 \phi_{h}, h-\bar{h}}\left(x_{B}\right)$, when the $Q^{2}$-dependence in $Q_{V}$ and in the FFs can be neglected, we obtain

$$
\begin{aligned}
A_{U U, d}^{\cos 2 \phi_{h}, h-\bar{h}}\left(x_{B}\right)= & \hat{\Phi}\left(x_{B}\right)\left\{\mathcal{N}_{\mathrm{BM}}^{Q_{V}}\left(x_{B}\right) \hat{C}_{\mathrm{BM}}^{h}\right. \\
& \left.+\frac{M M_{d}}{\langle Q\rangle^{2}} \hat{C}_{\mathrm{Cahn}}^{h}\right\}, \\
h & =\pi^{+}, K^{+}, h^{+},
\end{aligned}
$$

where $\hat{\Phi}\left(x_{B}\right)$ is a completely fixed kinematic function, the same for all final hadrons:

$$
\hat{\Phi}\left(x_{B}\right)=\frac{2(1-\bar{y})}{\left[1+(1-\bar{y})^{2}\right]} .
$$

The contribution from the Cahn effect is of order $1 / Q^{2}$ compared to the BM contribution. The constants $\hat{C}_{\mathrm{BM}}^{h}$ and $\hat{C}_{\text {Cahn }}^{h}$ are:

$$
\begin{aligned}
\hat{C}_{\mathrm{BM}}^{h}= & -\frac{e K}{M_{\mathrm{BM}} M_{C}} \frac{\int d z_{h} z_{h}\left[\Delta^{N} D_{q_{V} \uparrow}^{h}\left(z_{h}\right)\right] /\left\langle P_{T}^{2}\right\rangle_{\mathrm{BM}}}{\int d z_{h}\left[D_{q_{V}}^{h}\left(z_{h}\right)\right]}, \\
K \equiv & \frac{\left\langle k_{\perp}^{2}\right\rangle_{\mathrm{BM}}^{2}\left\langle p_{\perp}^{2}\right\rangle_{C}^{2}}{\left\langle k_{\perp}^{2}\right\rangle\left\langle p_{\perp}^{2}\right\rangle} \\
\hat{C}_{\mathrm{Cahn}}^{h}= & \frac{1}{2 M M_{d}\left\langle k_{\perp}^{2}\right\rangle\left\langle p_{\perp}^{2}\right\rangle} \frac{\int d z_{h}\left[D_{q_{V}}^{h}\left(z_{h}\right)\right] J\left(z_{h}\right)}{\int d z_{h}\left[D_{q_{V}}^{h}\left(z_{h}\right)\right]} \\
J\left(z_{h}\right) \equiv & \int d P_{T}^{2} e^{-\frac{P_{T}^{2}}{\left\langle p_{\perp}^{2}\right\rangle}} \int d k_{\perp}^{2} k_{\perp}^{2} e^{-k_{\perp\left\langle\frac{\left\langle P_{\perp}\right.}{2}\right\rangle\left\langle p_{\perp}^{2}\right\rangle}} \\
& \times \int_{0}^{2 \pi} d \phi \cos 2 \phi e^{a \cos \phi}, \\
a= & \frac{2 z_{h} k_{\perp} P_{T}}{\left\langle p_{\perp}^{2}\right\rangle} .
\end{aligned}
$$


(2) The Sivers asymmetry is given in (39).

(3) The assumed relation (27) between the BM and Sivers functions leads to the following relation between the $x_{B}$-dependent azimuthal $\cos 2 \phi_{h}$ asymmetry $A_{U U}^{\cos 2 \phi_{h}} \equiv\left\langle\cos 2 \phi_{h}\right\rangle$ and the Sivers asymmetry on a deuteron target:

$$
\begin{aligned}
& A_{U U, d}^{\cos 2 \phi_{h}, h-\bar{h}}\left(x_{B}\right)-\hat{C}_{\overparen{B M}}^{h} \hat{\Phi}\left(x_{B}\right) A_{U T, d}^{\mathrm{Siv}, h-\bar{h}}\left(x_{B}\right) \\
& =\frac{M M_{d}}{\langle Q\rangle^{2}} \hat{\Phi}\left(x_{B}\right) \hat{C}_{\text {Cahn }}^{h}, \quad h=\pi^{+}, K^{+}, h^{+} .
\end{aligned}
$$

This relation and Eq. (45) were previously obtained in [2] without including the $1 / Q^{2}$-Cahn contribution. However, as present measurements are performed at rather low $Q^{2}$, now we have included the $1 / Q^{2}$-suppressed Cahn contribution as well. This is important for comparing to existing data, which we shall do in the next section.

The constants $\hat{C}_{\widehat{\mathrm{BM}}}^{h}$ is expressed in terms of the parameter $\lambda_{Q_{V}}$ and the TMD-fragmentation functions:

$$
\begin{aligned}
\hat{C}_{\mathrm{BM}}^{h}= & \lambda_{Q_{V}} \frac{-2 \sqrt{2 e}}{\sqrt{\pi}} \frac{\left\langle p_{\perp}^{2}\right\rangle_{C}^{2}}{M_{C}\left\langle p_{\perp}^{2}\right\rangle} \frac{\int d z_{h} z_{h}\left[\Delta^{N} D_{q_{V} \uparrow}^{h}\left(z_{h}\right)\right] /\left\langle P_{T}^{2}\right\rangle_{\overparen{\mathrm{BM}}}}{\int d z_{h} z_{h}\left[D_{q_{V}}^{h}\left(z_{h}\right)\right] / \sqrt{\left\langle P_{T}^{2}\right\rangle_{S}}} \\
& h=\pi^{+}, K^{+}, h^{+} .
\end{aligned}
$$

The FFs $\left[D_{q_{V}}^{h^{+}}\left(z_{h}\right)\right]$ and $\left[\Delta^{N} D_{q_{V} \uparrow}^{h^{+}}\left(z_{h}\right)\right]$ are given in Eqs. (35)-(38).

The relations (42) and (50) between the Sivers $A_{U T, d}^{\mathrm{Siv}, h-\bar{h}}\left(x_{B}\right)$ and the unpolarized azimuthal $A_{U U, d}^{\cos \phi_{h}, h-\bar{h}}\left(x_{B}\right)$ or $A_{U U, d}^{\cos 2 \phi_{h}, h-\bar{h}}\left(x_{B}\right)$ asymmetries, in which $C_{i}^{h}$, respectively $\hat{C}_{i}^{h}$, are parameters, represent the following:

(1) two independent direct tests of the assumed relation (27) between the BM and Sivers functions, in which only measurable quantities enter, and no knowledge about the TMD functions is required and,

(2) two independent ways for extracting the Cahn contribution from data.

\section{TESTS USING THE COMPASS DATA FOR $\boldsymbol{h}^{ \pm}$ PRODUCTION ON A DEUTERIUM TARGET}

Here we test relation (27) using the COMPASS SIDIS data on deuteron for production of charged hadrons $h^{ \pm}$for the spin averaged angular distributions $A_{U U}^{\cos \phi_{h}, h^{ \pm}}\left(x_{B}\right)$ and $A_{U U}^{\cos 2 \phi_{h}, h^{ \pm}}\left(x_{B}\right)$ [20], and the single-spin Sivers asymmetry data $A_{U T}^{\mathrm{Siv}, h^{ \pm}}\left(x_{B}\right)$ [21]. We perform the fits in three steps.

First, we form the difference asymmetries $A_{J}^{h^{+}-h^{-}}, J=$ $\left\langle\cos \phi_{h}\right\rangle,\left\langle\cos 2 \phi_{h}\right\rangle$, Siv from the corresponding usual asymmetries $A_{j}^{h^{+}}$and $A_{j}^{h^{-}}$for positive and negative charged hadron production [6]:

$$
\begin{aligned}
A_{J}^{h^{+}-h^{-}} & =\frac{1}{1-r}\left(A_{J}^{h^{+}}-r A_{J}^{h^{-}}\right), \\
J & =\left\langle\cos \phi_{h}\right\rangle,\left\langle\cos 2 \phi_{h}\right\rangle, \text { Siv } .
\end{aligned}
$$

Here $r$ is the ratio of the unpolarized $x_{B}$-dependent SIDIS cross sections for production of negative and positive hadrons $r=\sigma^{h^{-}}\left(x_{B}\right) / \sigma^{h^{+}}\left(x_{B}\right)$ measured in the same kinematics [6]. As the available data for the different asymmetries is in different $x_{B}$ bins, which do not match we need to interpolate the data. It turns out that a linear interpolation is adequate. Hereafter we work with the interpolation functions $A_{J}^{h^{ \pm}}\left(x_{B}\right)$ only.

When we determine the errors of the difference asymmetries we assume that data is not correlated.

Second, we choose the $Q^{2}$ interval where the $Q^{2}$ dependence of the collinear PDF's and FFs can be neglected. In the COMPASS kinematics to each value of $\left\langle Q^{2}\right\rangle$ corresponds one definite value of $\left\langle x_{B}\right\rangle$, thus fixing the $Q^{2}$ interval we fix also the $x_{B}$-interval. Using the available CTEQ parametrizations for the PDFs [22], we see that there is almost no $Q^{2}$ dependence in the valence-quark distributions $u_{V}$ and $d_{V}$ in the whole $Q^{2}$-range covered by COMPASS, $Q^{2} \simeq[1-17] \mathrm{GeV}^{2}$, i.e. in the whole $x_{B}$ interval. To get some feeling for the $Q^{2}$ dependence of the fragmentation function $D_{u_{V}}^{h^{+}}$to charged hadrons, bearing in mind that $h^{ \pm}$ production is strongly dominated by $\pi^{ \pm}$production, in Fig. 1 we plot the dependence of $D_{u_{V}}^{\pi^{+}}$on $Q^{2}$ for different values of $z_{h}$. We use the parametrization in [23] obtained using the recent HERMES [24] and preliminary COMPASS data [25] on multiplicities. This parametrization is in qualitative agreement with the one obtained from analysis of the latest COMPASS data [26]. We see that, aside from the small values of $Q^{2} \lesssim 1.8 \mathrm{GeV}^{2}$, the $Q^{2}$ dependence is weak. We thus consider it reasonably safe to use the following fitting interval $x_{B} \in[0.014,0.13]$ corresponding to $Q^{2} \in[1.77,16.27] \mathrm{GeV}^{2}$.

Third, we fit the parameters in Eqs. (42) and (50) using $\chi^{2}$ analysis. There are two ways to utilize (42) and (50), we shall follow both of them:

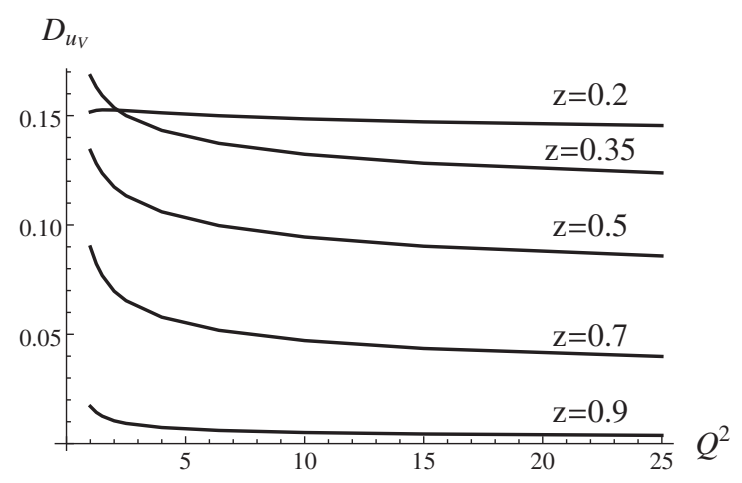

FIG. 1. The dependence of $D_{u_{V}}^{\pi^{+}}$on $Q^{2}$ for different values of $z_{h}=0.2,0.3,0.5,0.7,0.9$. 
$(\mathcal{A})$ Provided there is enough data, we consider both $C_{\text {Cahn }}^{h}$ and $C_{\overparen{B M}}^{h}$ (respectively $\hat{C}_{\text {Cahn }}^{h}$ and $\hat{C}_{\overparen{B M}}^{h}$ ) as fitted parameters.

$(\mathcal{B})$ Alternatively, first we calculate the Cahn constants, $C_{\text {Cahn }}^{h}$ or $\hat{C}_{\text {Cahn }}^{h}$, using the obtained expressions (30), (48), and then fit the same data with just a single free parameter, $C_{\overparen{B M}}^{h}$ or $\hat{C}_{\overparen{B M}}^{h}$. The problem with this approach, however, is that the Cahn constants depend both on the chosen parametrizations for the FFs, which don't differ so much, and on the values of the parameters $\left\langle k_{\perp}^{2}\right\rangle,\left\langle p_{\perp}^{2}\right\rangle$, which, as discussed in Sec. II B, are rather poorly known and vary considerably. Consequently the main interest in this second approach will be to compare the calculated Cahn constants with those determined by fitting the parameters as in $(\mathcal{A})$ above.

The used $\chi^{2}$ for the $\left\langle\cos \phi_{h}\right\rangle^{h^{ \pm}}$and $\left\langle\cos 2 \phi_{h}\right\rangle^{h^{ \pm}}$asymmetries are:

$$
\begin{aligned}
\chi_{\cos \phi}^{2} & =\int_{x_{i}}^{x_{f}} d x \frac{\left[F_{\exp }(x)-F_{T H}(x)\right]^{2}}{\left[\Delta F_{\exp }(x)\right]^{2}}, \\
\chi_{\cos 2 \phi}^{2} & =\int_{x_{i}}^{x_{f}} d x \frac{\left[\hat{F}_{\exp }(x)-\hat{F}_{T H}(x)\right]^{2}}{\left[\Delta \hat{F}_{\exp }(x)\right]^{2}}
\end{aligned}
$$

which take into account the different widths of $x_{B}$-bins in which the data is collected. Here $F_{\exp }\left(x_{B}\right)$ and $\hat{F}_{\exp }\left(x_{B}\right)$ denote the proper combinations of experimental data-the 1.h.s. of Eqs. (42) and (50), while $F_{T H}\left(x_{B}\right)$ and $\hat{F}_{T H}\left(x_{B}\right)$ are the corresponding theoretical expressions-the r.h.s. of (42) and (50):

$$
\begin{aligned}
& F_{\exp }(x)=A_{U U, d}^{\cos \phi_{h}, h^{+}-h^{-}}\left(x_{B}\right)-C_{\overparen{B M}}^{h} \Phi\left(x_{B}\right) A_{U T, d}^{\mathrm{Siv}, h^{+}-h^{-}}\left(x_{B}\right), \\
& F_{T H}(x)=C_{\mathrm{Cahn}}^{h} \Phi(x) \\
& \hat{F}_{\exp }(x)=A_{U U, d}^{\cos 2 \phi_{h}, h^{+}-h^{-}}\left(x_{B}\right)-\hat{C}_{\overparen{\mathrm{BM}}}^{h} \hat{\Phi}\left(x_{B}\right) A_{U T, d}^{\mathrm{Siv}, h^{+}-h^{-}}\left(x_{B}\right), \\
& \hat{F}_{T H}(x)=\frac{M M_{d}}{\langle Q\rangle^{2}\left(x_{B}\right)} \hat{C}_{\mathrm{Cahn}}^{h} \hat{\Phi}\left(x_{B}\right)
\end{aligned}
$$

In this way the tested relations are put in the standard form "experiment" = "theory". Note however, that the situation here is rather peculiar because the errors of experimental data $\Delta F_{\exp }\left(x_{B}\right)$ and $\Delta \hat{F}_{\exp }\left(x_{B}\right)$ contain not only the errors of the asymmetries $\Delta A_{U U, d}^{\cos \phi_{h}}, \Delta A_{U U, d}^{\cos 2 \phi_{h}}$ and $\Delta A_{U U, d}^{\mathrm{Siv}}$, but the fitting parameter as well. We have:

$$
\begin{gathered}
\Delta F_{\exp }\left(x_{B}\right)=\sqrt{\left(\Delta A_{U U, d}^{\cos \phi_{h}, h^{+}-h^{-}}\left(x_{B}\right)\right)^{2}+\left(C_{\overparen{\mathrm{BM}}}^{h}\right)^{2} \Phi^{2}\left(x_{B}\right)\left(\Delta A_{U T, d}^{\mathrm{Siv}, h^{+}-h^{-}}\left(x_{B}\right)\right)^{2}} \\
\Delta \hat{F}_{\exp }\left(x_{B}\right)=\sqrt{\left(\Delta A_{U U, d}^{\cos 2 \phi_{h}, h^{+}-h^{-}}\left(x_{B}\right)\right)^{2}+\left(\hat{C}_{\widetilde{\mathrm{BM}}}^{h}\right)^{2} \hat{\Phi}^{2}\left(x_{B}\right)\left(\Delta A_{U T, d}^{\mathrm{Siv}, h^{+}-h^{-}}\left(x_{B}\right)\right)^{2}} .
\end{gathered}
$$

In (53) the upper limit $x_{f}=0.13$ is fixed by the existing data for both $A_{U U, d}^{\cos \phi_{h}}$ and $A_{U U, d}^{\cos 2 \phi_{h}}$ asymmetries, and $x_{i}$ is determined by the requirement that it is safe to ignore $Q^{2}$ variation.

To test quantitatively the applicability of Eqs. (42) and (50) for small $x_{B}$ we have made series of fits with increasing $x_{i}$ starting with $x_{i(\min )}=0.006$ and going up to $x_{i(\max )}=0.025$ and we introduce the quantity $\chi^{2} / \Delta x$, which is $\chi^{2}$ normalized to the length of the fitting interval $\Delta x=x_{f}-x_{i}$. (It is the continuum analogue of $\chi^{2}$ per degree of freedom in the discrete case.) The obtained $\chi^{2} / \Delta x\left(x_{i}\right)$ functions for both asymmetries are plotted on Fig. 2. Both of them exhibit a step-like behavior with the step at roughly the same position about $x_{B}=0.014$. This shows that Eqs. (42), (50) hold only for $x_{B} \geq 0.014$.

In the next two subsections, we present the obtained values and standard deviations of the fitted parameters. The values correspond to the best fit of the available data with $\chi^{2}$ defined as above. We use Monte Carlo simulation in order to estimate the deviations of the fitting parameters. On the basis of the experimental data and assuming they have a Gaussian distribution we construct $10^{3}$ sets of "virtual experimental data". For each virtual experimental data set we determine corresponding best-fit parameters. Thus we obtain for each parameter $C_{\overparen{\mathrm{BM}}}^{h}, C_{\mathrm{Cahn}}^{h}, \hat{C}_{\overparen{\mathrm{BM}}}^{h}$ and $\hat{C}_{\text {Cahn }}^{h}$ a set with $10^{3}$ data values. Further, we filtered out the data values which are attracted by the false local minimum corresponding not to small $\left(F_{\exp }(x)-F_{T H}(x)\right)^{2}$ or $\left(\hat{F}_{\exp }(x)-\hat{F}_{T H}(x)\right)^{2}$ but to large $\Delta F_{\exp }\left(x_{B}\right)$ [respectively, $\left.\Delta \hat{F}_{\exp }\left(x_{B}\right)\right]$. In this way we end up with four Gaussian distributed sets for the parameters $C_{\overparen{\mathrm{BM}}}^{h}, C_{\mathrm{Cahn}}^{h}, \hat{C}_{\overparen{\mathrm{BM}}}^{h}$ and $\hat{C}_{\text {Cahn }}^{h}$, we calculate the standard deviation for each of them and report it as the parameter error.

\section{A. Test using the COMPASS data on $A_{U U}^{\cos \phi_{h}}$}

The difference asymmetries $A_{U U}^{\cos \phi_{h}, h^{+}-h^{-}}$and $A_{U T}^{\operatorname{Siv}, h^{+}-h^{-}}$ are presented on Fig. 3, panel (a). Note that the Sivers asymmetry $A_{U T, d}^{\mathrm{Siv}, h^{+}-h^{-}}\left(x_{B}\right)$ is almost zero and rather poorly determined, which suggests, and is proven in our fits, that the corresponding fitting parameter $C_{\overparen{\mathrm{BM}}}^{h}$ will be poorly determined. 


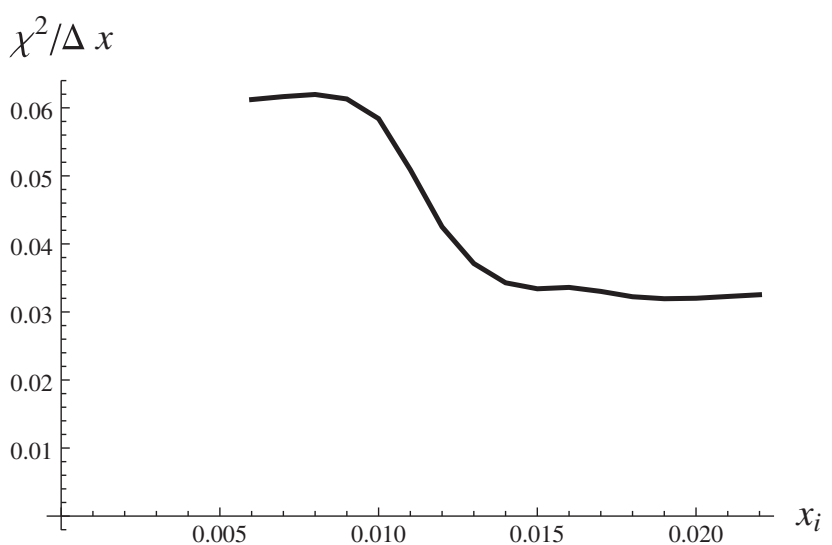

(a)

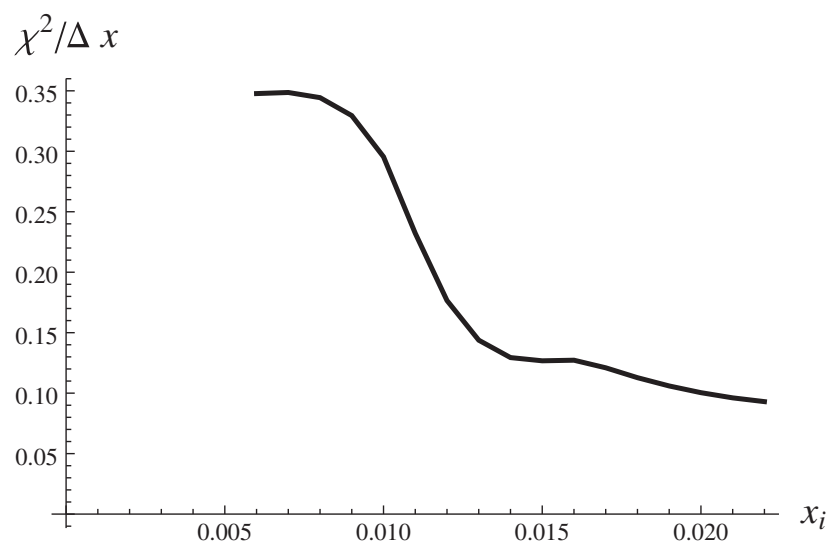

(b)

FIG. 2. The quality of the fit $\chi^{2} / \Delta x$ as a function of $x_{i}$ with $\Delta x=x_{f}-x_{i}$. Panel (a) is for $\left\langle\cos \left(\phi_{h}\right)\right\rangle$ asymmetry, both $C_{\mathrm{Cahn}}$ and $C_{\widetilde{\mathrm{BM}}}$ are fitted. Panel (b) is for $\left\langle\cos \left(2 \phi_{h}\right)\right\rangle$ asymmetry, both $\hat{C}_{\text {Cahn }}$ and $\hat{C}_{\widetilde{B M}}$ are fitted. Note the different scales in the two panels.

(i) $(\mathcal{A})$ The results of our fit in approach $(\mathcal{A})$, when both $C_{\text {Cahn }}^{h}$ and $C_{\overparen{B M}}^{h}$ are fitted, are presented on panels (b), (c) and (d) of Fig. 3 for the three $x_{B^{-}}$ intervals: b) $x_{B} \gtrsim 0.006\left(Q^{2} \geq 1.15 \mathrm{GeV}^{2}\right)$, c) for $x_{B} \gtrsim 0.014\left(Q^{2} \geq 1.77 \mathrm{GeV}^{2}\right)$ and $\left.\mathrm{d}\right) x_{B} \gtrsim 0.022$
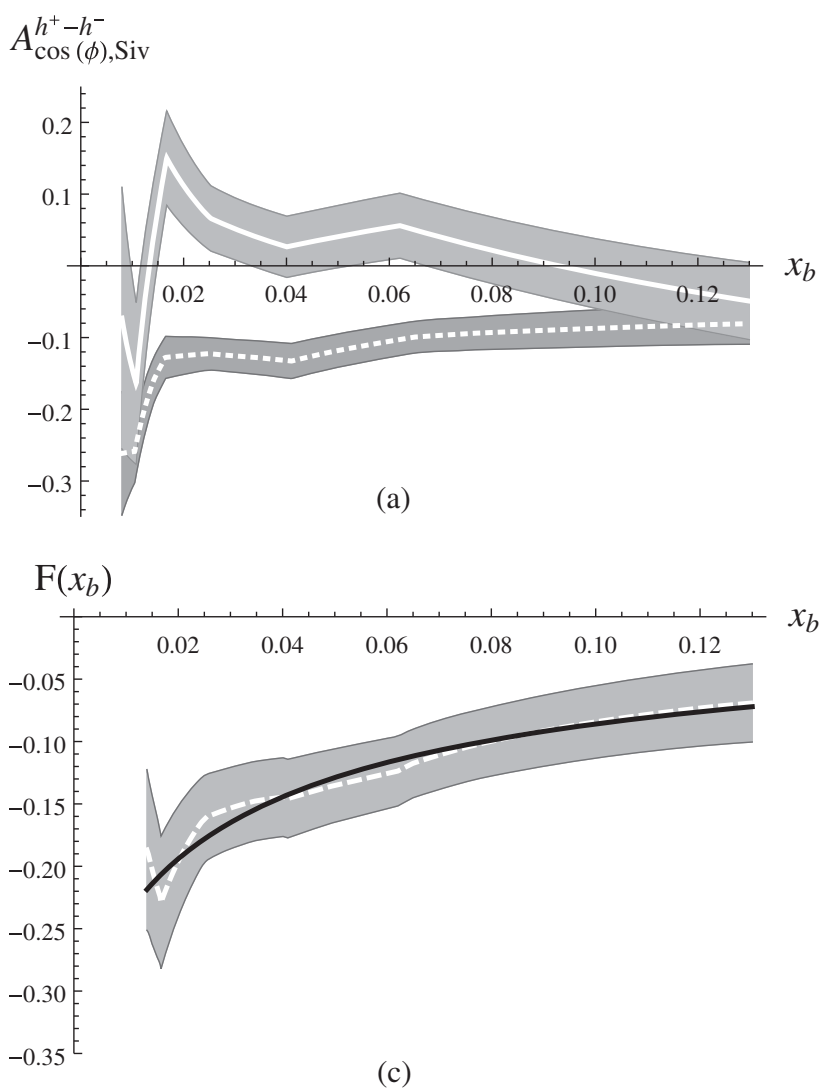

$\left(Q^{2} \geq 2.43 \mathrm{GeV}^{2}\right)$. Panels (c) and (d) show that relation (27) holds for $x_{B} \gtrsim 0.014$, while the discrepancy between experiment and theory in case (b) shows that at small $x_{B} \lesssim 0.014$ relation (27) does not hold. This agrees with the results of Fig. 2(a).
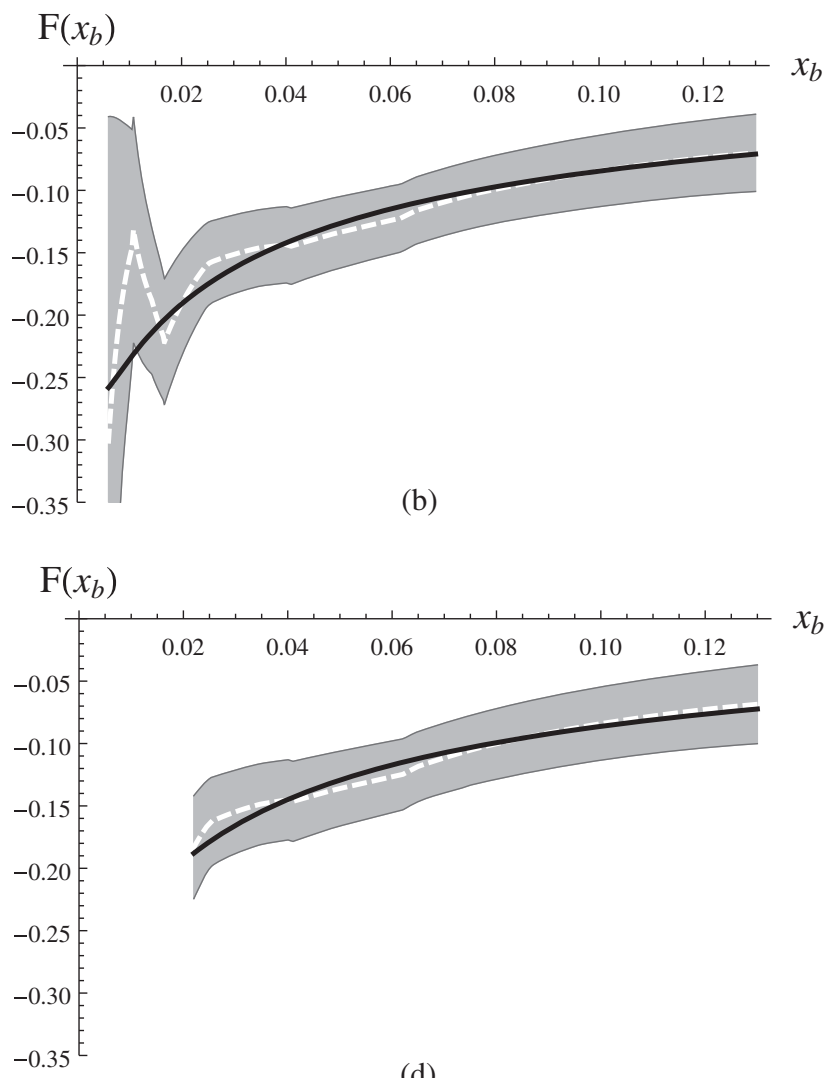

(d)

FIG. 3. The results of our tests using $A_{U U, d}^{\cos \phi_{h}}\left(x_{B}\right)$, Eq. (42), following approach $(\mathcal{A})$ : On panel (a) are the used asymmetries $A_{U T, d}^{\mathrm{Siv}, h^{+}-h^{-}}\left(x_{B}\right)$ (white solid line) and $A_{U U, d}^{\cos \phi_{h}, h^{+}-h^{-}}\left(x_{B}\right)$ (white dashed line) with their statistical errors. On panels (b), (c) and (d) are our fits with different $x_{i}$ : (b) $x_{B} \gtrsim 0.006$, (c) $x_{B} \gtrsim 0.014$ and (d) $x_{B} \gtrsim 0.022$; the dashed white lines are $F_{\exp }\left(x_{B}\right)$ with their errors as shaded corridors, the solid black lines are $F_{T H}\left(x_{B}\right)$. 
(ii) $(\mathcal{B})$ In approach $(\mathcal{B})$ we need an expression for $C_{\text {Cahn }}^{h}$ with integration over the measured $P_{T}$ interval in COMPASS:

$$
\begin{aligned}
C_{\mathrm{Cahn}}^{h}= & -\frac{2}{\sqrt{\pi}}\left\langle k_{\perp}^{2}\right\rangle \\
& \times \frac{\int d z_{h} z_{h} \int d P_{T} P_{T}^{2} e^{-P_{T}^{2} /\left\langle P_{T}^{2}\right\rangle}\left[D_{q_{V}}^{h}\left(z_{h}\right)\right] /\left\langle P_{T}^{2}\right\rangle^{2}}{\int d z_{h} \int d P_{T} P_{T} e^{-P_{T}^{2} /\left\langle P_{T}^{2}\right\rangle}\left[D_{q_{V}}^{h}\left(z_{h}\right)\right] /\left\langle P_{T}^{2}\right\rangle},
\end{aligned}
$$

where the limits of integration are $\left[P_{T, \min }, P_{T, \max }\right]=$ $[0.1,1.0 \mathrm{GeV}]$ and $z_{h}=[0.2,0.85]$ [20]. (If the integration over $P_{T}$ is in the interval $P_{T} \in[0, \infty]$ we recover Eq. (30).)

We need also the FF for unidentified charged hadrons $\left[D_{q_{V}}^{h^{+}}\right]$. To estimate this, we neglect the contribution from produced protons, (about $1 \%$ ) and use:

$$
\begin{aligned}
{\left[D_{q_{V}}^{h^{+}}\right] \equiv e_{u}^{2} D_{u_{V}}^{h^{+}}+e_{d}^{2} D_{d_{V}}^{h^{+}} } & =\left(e_{u}^{2}-e_{d}^{2}\right) D_{u_{V}}^{\pi^{+}}+e_{u}^{2} D_{u_{V}}^{K^{+}}, \\
D_{q_{V}}^{h} & =D_{q}^{h}-D_{\bar{q}}^{h},
\end{aligned}
$$

where we have used SU(2)-invariance for the pions, implying:

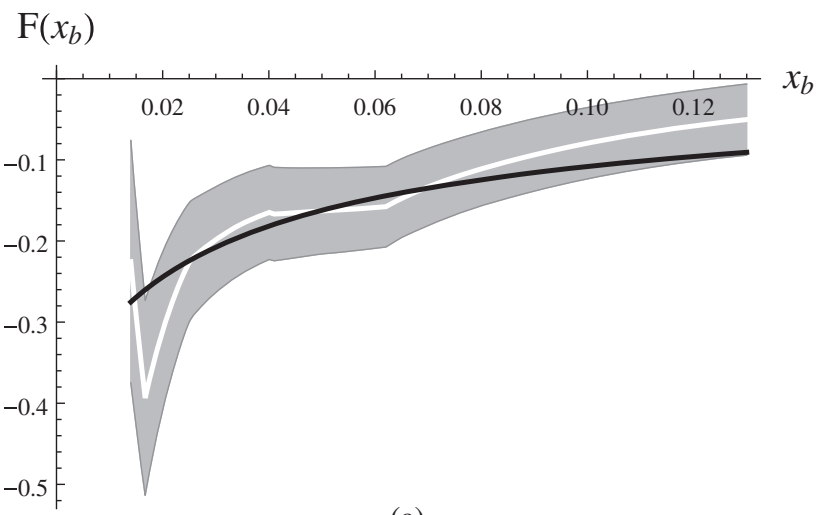

(a)

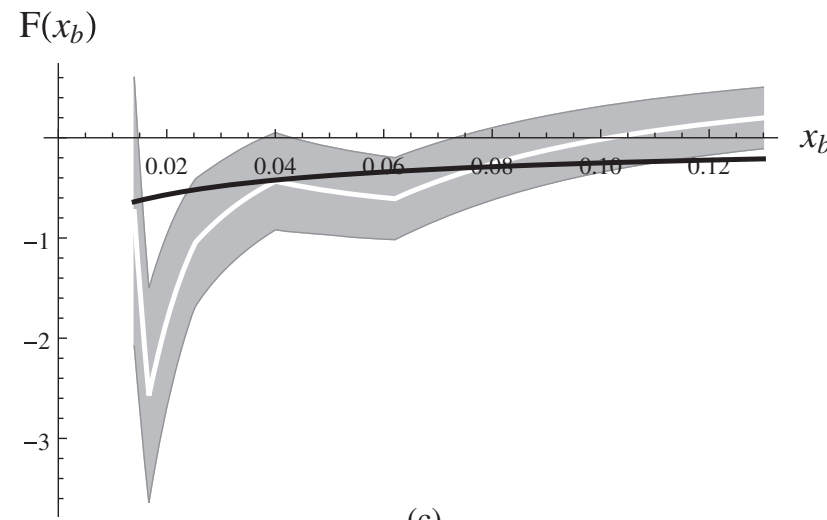

(c)

$$
D_{u_{V}}^{\pi^{+}}=-D_{d_{V}}^{\pi^{+}},
$$

and $D_{d_{V}}^{K^{+}}=0$, which follows from the quark content of kaons; this assumption is used in all present analyses in extracting the kaon FFs.

We use two of the available parametrizations for the FFs: AKK [27] and LSS [23] and find that the value of $C_{\text {Cahn }}^{h}$ is not sensitive to the used parametrization; also, as expected, it is not sensitive to the chosen $\left\langle Q^{2}\right\rangle$. However it is very sensitive to the values $\left\langle k_{\perp}^{2}\right\rangle$ and $\left\langle p_{\perp}^{2}\right\rangle$. We find that the quality of the fits in the approach $\mathcal{B}$, with one exception, are considerably worse than in the approach $\mathcal{A}$ when both $C_{\text {Cahn }}^{h}$ and $C_{\overparen{B M}}^{h}$ are fitted-see Fig. 4. The exception is for the values $\left\langle k_{\perp}^{2}\right\rangle=0.18,\left\langle p_{\perp}^{2}\right\rangle=0.20 \mathrm{GeV}^{2}$, where the calculated $C_{\text {Cahn }}^{h}$ coincides with the fitted value. In the case of $\left\langle k_{\perp}^{2}\right\rangle=0.25,\left\langle p_{\perp}^{2}\right\rangle=0.20 \mathrm{GeV}^{2}$ the calculated $C_{\text {Cahn }}^{h}$ is within the error of the fitted one.

For $\left\langle k_{\perp}^{2}\right\rangle=0.57,\left\langle p_{\perp}^{2}\right\rangle=0.12 \mathrm{GeV}^{2}$ the discrepancy between calculated and fitted $C_{\mathrm{Cahn}}^{h}$ is $4.9 \sigma$ and goes up to $5.8 \sigma$ for $C_{\overparen{B M}}^{h}$. For $\left\langle k_{\perp}^{2}\right\rangle=0.61,\left\langle p_{\perp}^{2}\right\rangle=0.19 \mathrm{GeV}^{2}$ the discrepancy is $2.1 \sigma$ for $C_{\text {Cahn }}^{h}$ and $2.6 \sigma$ for $C_{\overparen{B M}}^{h}$.

This can be verified also in Table I, where the obtained numerical values for $C_{\text {Cahn }}^{h}$ and $C_{\mathrm{BM}}^{h}$ in approaches $(\mathcal{A})$ and

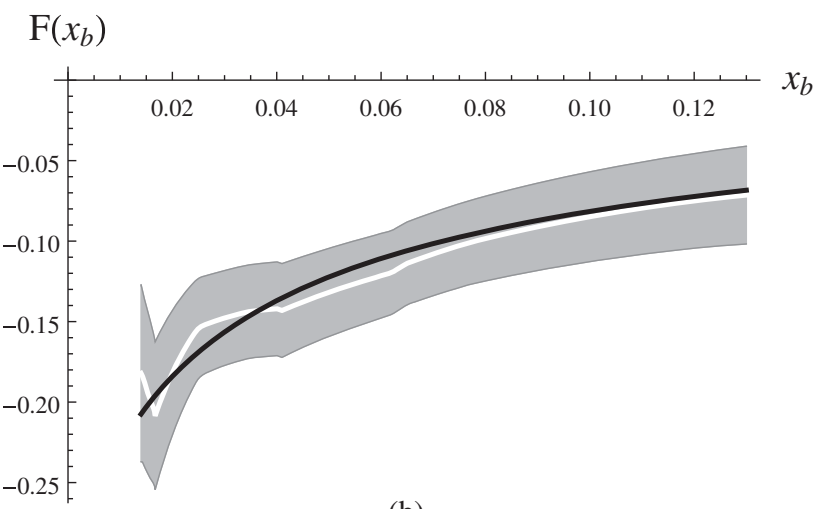

(b)

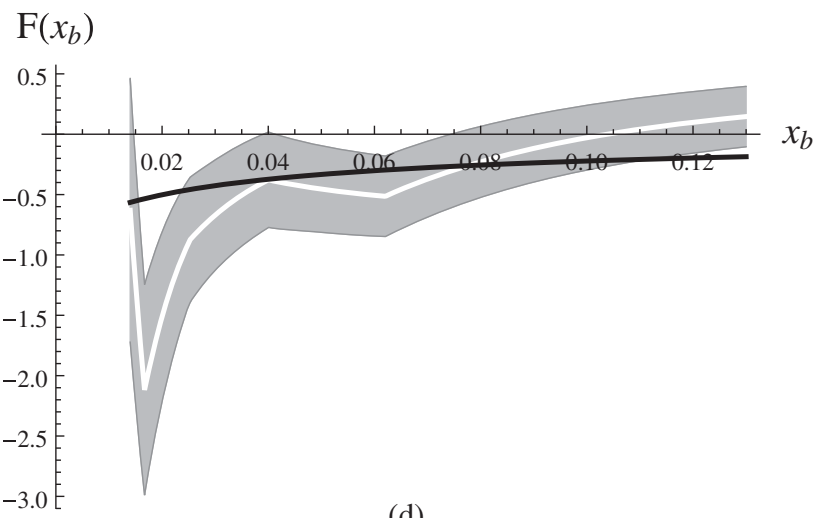

(d)

FIG. 4. The fits corresponding to approach $(\mathcal{B})$, Table I. The white line is $F_{\text {exp }}\left(x_{B}\right)$ and the black one is $F_{T H}\left(x_{B}\right)$ with $C_{\text {Cahn }}$ calculated with different values of $\left\langle k_{\perp}^{2}\right\rangle$ and $\left\langle p_{\perp}^{2}\right\rangle$. Panel (a) is for $\left\langle k_{\perp}^{2}\right\rangle=0.25\left\langle p_{\perp}^{2}\right\rangle=0.20\left[\mathrm{GeV}^{2}\right]$, (b) is for $\left\langle k_{\perp}^{2}\right\rangle=0.18\left\langle p_{\perp}^{2}\right\rangle=0.20\left[\mathrm{GeV}^{2}\right]$, (c) is for $\left\langle k_{\perp}^{2}\right\rangle=0.57\left\langle p_{\perp}^{2}\right\rangle=0.12\left[\mathrm{GeV}^{2}\right]$ and (d) is for $\left\langle k_{\perp}^{2}\right\rangle=0.61\left\langle p_{\perp}^{2}\right\rangle=0.19\left[\mathrm{GeV}^{2}\right]$. Note the different scales in the panels. 
TABLE I. The numerical values for the parameters: $(\mathcal{A})$ : Both $C_{\text {Cahn }}^{h}$ and $C_{\widetilde{B M}}^{h}$ are obtained fitting Eq. (42), the errors (which correspond to one standard deviation) are obtained with MC simulation. (B)): $C_{\text {Cahn }}^{h}{ }_{1 \mathrm{SM}}^{\mathrm{BM}}$ calculated using Eq. (57) in which the FFs are from LSS [23], and the values for $\left\langle k_{\perp}^{2}\right\rangle$ and $\left\langle p_{\perp}^{2}\right\rangle$ are those discussed in Sec. II B. $C_{\text {BM }}^{h}$ is obtained fitting Eq. (42). We give two errors. First we give the error due to momenta spread (only given for the cases where errors of $\left\langle k_{\perp}^{2}\right\rangle,\left\langle p_{\perp}^{2}\right\rangle$ are known). Second, in parentheses, we give the total standard deviation due to both momenta and data errors.

\begin{tabular}{lcccrr}
\hline \hline & $\mathcal{A}$ & \multicolumn{3}{c}{$\mathcal{B}$} \\
\hline$\left\langle k_{\perp}^{2}\right\rangle\left[\mathrm{GeV}^{2}\right]$ & & 0.25 & 0.18 & $0.57 \pm 0.08$ & $0.61 \pm 0.20$ \\
$\left\langle p_{\perp}^{2}\right\rangle\left[\mathrm{GeV}^{2}\right]$ & -0.20 & 0.20 & $0.12 \pm 0.01$ & $0.19 \pm 0.02$ \\
$C_{\text {Cahn }}^{h}$ & $-0.167 \pm 0.043$ & -0.21 & -0.16 & $-0.49 \pm 0.05$ & $-0.43 \pm 0.10$ \\
$C_{\overparen{\mathrm{BM}}}^{h}$ & $0.55 \pm 0.80$ & $1.43( \pm 1.7)$ & $0.44( \pm 0.93)$ & $13 \pm 2( \pm 6)$ & $11 \pm 4( \pm 6)$ \\
$\chi^{2} / \Delta x$ & 0.034 & 0.27 & 0.055 & 0.58 & 0.57 \\
\hline \hline
\end{tabular}

$(\mathcal{B})$ are presented. The presented errors correspond to 1 standard deviation. Note that from the analytic expression Eq. (30), it follows that $C_{\text {Cahn }}^{h}$ should be negative, which is in agreement with the value obtained from the fit.

To the best of our knowledge, this is the first time that the Cahn contribution $C_{\text {Cahn }}^{h}$ has been determined from data and it is puzzling that its value is in agreement with a calculated result based on the early values of the Gaussian

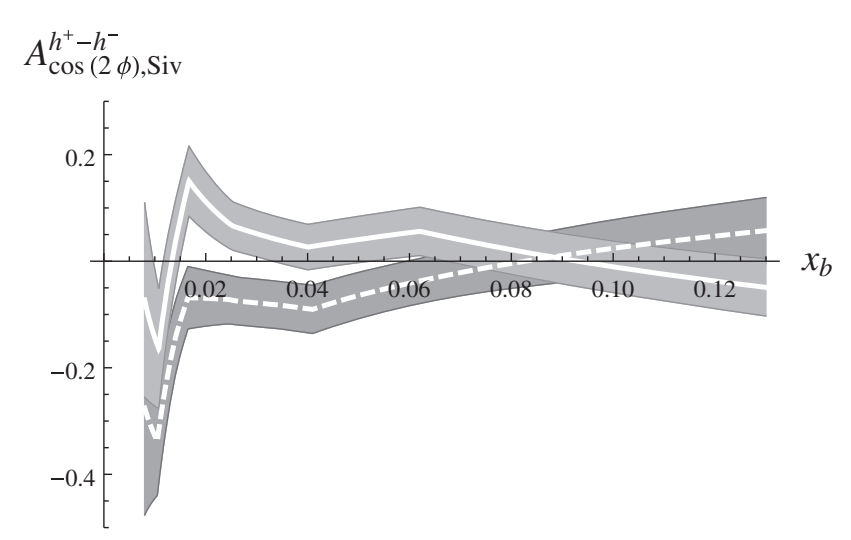

(a)

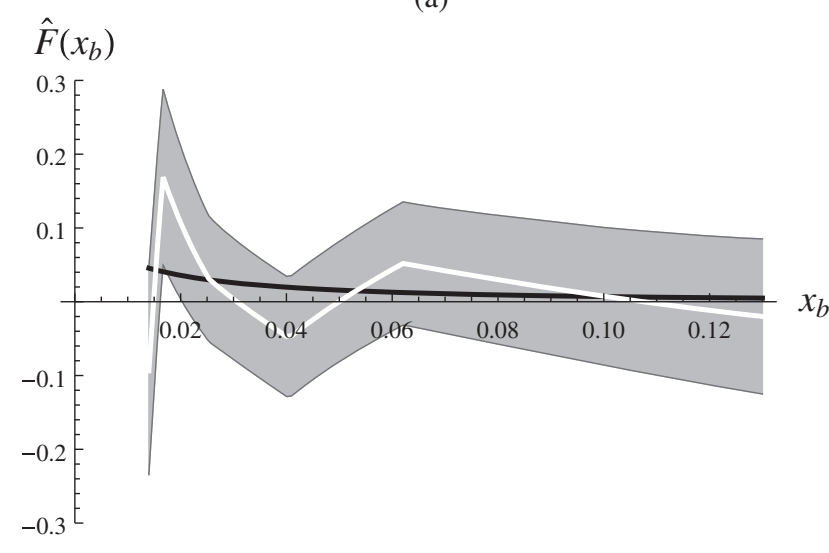

(c) parameters $\left\langle k_{\perp}^{2}\right\rangle=0.18,\left\langle p_{\perp}^{2}\right\rangle=0.20 \mathrm{GeV}^{2}$, which are supposed to be ruled out by later measurements.

\section{B. Test using the COMPASS data on $A_{U U}^{\cos 2 \phi_{h}}$}

The used difference asymmetries $A_{U U}^{\cos 2 \phi_{h}, h^{+}-h^{-}}$and $A_{U T}^{\mathrm{Siv}, h^{+}-h^{-}}$are presented in Fig. 5a. Note that now both asymmetries are poorly determined with large relative

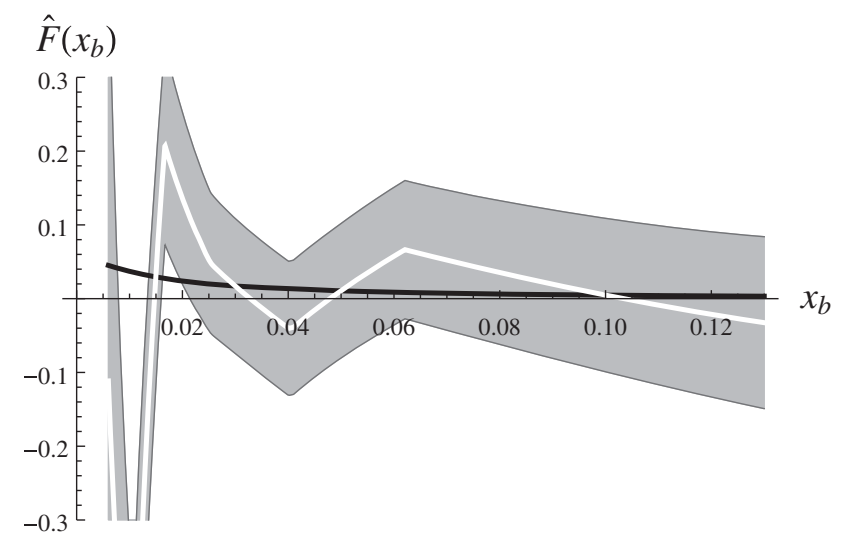

(b)

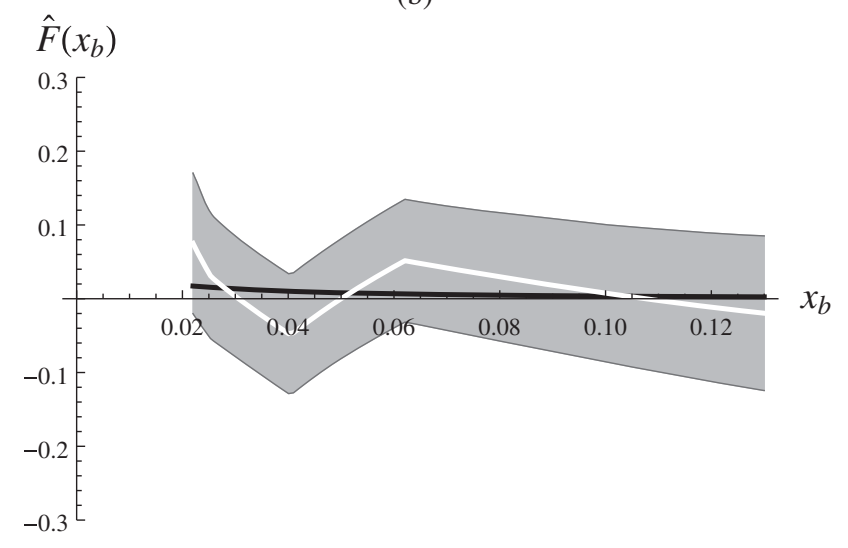

(d)

FIG. 5. The results of our tests using $A_{U U, d}^{\cos 2 \phi_{h}}\left(x_{B}\right)$, Eq. (50), following approach $(\mathcal{A})$ : On panel (a) are the used asymmetries $A_{U T, d}^{\text {Siv, } h^{+}-h^{-}}\left(x_{B}\right)$ (white solid line) and $A_{U U, d}^{\cos 2 \phi_{,}, h^{+}-h^{-}}\left(x_{B}\right)$ (white dashed line) with their statistical errors. On panels (b), (c) and (d) are our fits for different $x_{i}$ : (b) $x_{B} \gtrsim 0.006$, (c) $x_{B} \gtrsim 0.014$ and (d) $x_{B} \gtrsim 0.022$; the white lines are $\hat{F}_{\text {exp }}\left(x_{B}\right)$ with their errors as shaded corridors, the black lines are $\hat{F}_{T H}\left(x_{B}\right)$. 
TABLE II. The numerical values for the parameters: $(\mathcal{A})$ : Both $\hat{C}_{\text {Cahn }}^{h}$ and $\hat{C}_{\overparen{B M}}^{h}$ are obtained fitting Eq. (50), the errors (which correspond to 1 standard deviation) are obtained with MC simulation. $(\mathcal{B})$ : $\hat{C}_{\text {Cahn }}^{h}$ is calculated using Eq. (48) in which the FFs are from LSS [23], and the values for $\left\langle k_{\perp}^{2}\right\rangle$ and $\left\langle p_{\perp}^{2}\right\rangle$ are those discussed in Sec. II B. $\hat{C}_{\mathrm{BM}}^{h}$ is obtained fitting Eq. (50). We give two errors. First we give the error due to momenta spread (only given for the cases where errors of $\left\langle k_{\perp}^{2}\right\rangle,\left\langle p_{\perp}^{2}\right\rangle$ are known). Second, in parentheses, we give the total standard deviation due to both momenta and data errors.

\begin{tabular}{lccccc}
\hline \hline & $\mathcal{A}$ & \multicolumn{3}{c}{$\mathcal{B}$} \\
\hline$\left\langle k_{\perp}^{2}\right\rangle\left[\mathrm{GeV}^{2}\right]$ & & 0.25 & 0.18 & $0.57 \pm 0.08$ & $0.61 \pm 0.20$ \\
$\left\langle p_{\perp}^{2}\right\rangle\left[\mathrm{GeV}^{2}\right]$ & & 0.20 & 0.20 & $0.12 \pm 0.01$ & $0.19 \pm 0.02$ \\
$\hat{C}_{\mathrm{Cahn}}^{h}$ & $0.083 \pm 0.22$ & 0.079 & 0.045 & $0.41 \pm 0.08$ & $0.32 \pm 0.15$ \\
$\hat{C}_{\overparen{\mathrm{BM}}}^{h}$ & $-1.6 \pm 1.6$ & $-1.9( \pm 1.9)$ & $-1.6( \pm 1.4)$ & $-4.9 \pm 0.8( \pm 3.0)$ & $-4.0 \pm 1.5( \pm 3.0)$ \\
$\chi^{2} / \Delta x$ & 0.13 & 0.15 & 0.16 & 0.20 & 0.19 \\
\hline \hline
\end{tabular}

errors, which implies that both fitting parameters $\left(\hat{C}_{\overparen{B M}}^{h}\right.$ and $\hat{C}_{\text {Cahn }}^{h}$ ) will be poorly determined. In Fig. 5, we show the fit to Eq. (50) in approach $(\mathcal{A})$ : panel (b) is the fit for $x_{B} \gtrsim 0.006$. The interval of discrepancy between experiment and theory is clearly visible. Panels (c) and (d) are for fits corresponding to the kinematics of the right "plateau" of the $\chi^{2}$ function (Fig. 2b). Panels (c) and (d) are for $x_{B} \gtrsim$ 0.014 and $x_{B} \gtrsim 0.022$, respectively. Analogously as for the $\left\langle\cos \phi_{h}\right\rangle^{h^{ \pm}}$-case, the theoretical function is within the experimental margins for $x_{i} \gtrsim 0.014$, however the relative errors in the present case are considerably bigger. Note that in the range $x_{B}>0.014, A_{U U, d}^{\cos 2 \phi_{h}, h^{+}-h^{-}}$and $A_{U T, d}^{\mathrm{Siv}, h^{+}-h^{-}}$have opposite signs, which suggests a small contribution from the Cahn effect. This follows also from our theoretical formula Eq. (50) and is confirmed by the obtained numerical values for $\hat{C}_{\mathrm{Cahn}}^{h}$ and $\hat{C}_{\mathrm{BM}}^{h}$ summarized in Table II.

The results of approaches $(\mathcal{A})$ and $(\mathcal{B})$ are compared in Table II. The errors cited therein correspond to 1 standard deviation. As expected, $\hat{C}_{\overparen{\mathrm{BM}}}^{h}$ is very poorly determined.

Here the calculated and fitted values of $\hat{C}_{\text {Cahn }}^{h}$ agree for $\left\langle k_{\perp}^{2}\right\rangle=0.25,\left\langle p_{\perp}^{2}\right\rangle=0.20$ and $\left\langle k_{\perp}^{2}\right\rangle=0.18,\left\langle p_{\perp}^{2}\right\rangle=$ $0.20 \mathrm{GeV}^{2}$ - a result similar to the one found from the $\left\langle\cos \phi_{h}\right\rangle$ asymmetry. For $\left\langle k_{\perp}^{2}\right\rangle=0.57,\left\langle p_{\perp}^{2}\right\rangle=0.12 \mathrm{GeV}^{2}$ the discrepancy between calculated and fitted $\hat{C}_{\text {Cahn }}^{h}$ is $1.4 \sigma$ and it goes up to $1.8 \sigma$ for $\hat{C}_{\overparen{\mathrm{BM}}}^{h}$. For $\left\langle k_{\perp}^{2}\right\rangle=0.61,\left\langle p_{\perp}^{2}\right\rangle=$ $0.19 \mathrm{GeV}^{2}$ the discrepancy is $0.9 \sigma$ for $\hat{C}_{\text {Cahn }}^{h}$ and $1.1 \sigma$ for $\hat{C}_{\mathrm{BM}}^{h}$.

\section{Comparison to the existing published extraction of the $\mathrm{BM}$ functions $[3,4]$}

In this paper we have tested the assumption of proportionality of the BM and Sivers functions for the sum of valence quarks $Q_{V}=u_{V}+d_{V}$, [Eq. (27)]. However, in Refs. [3,4] the BM functions have been extracted from the $\cos 2 \phi$ asymmetry assuming proportionality for each quark and antiquark flavor $q$ separately:

$$
\Delta f_{\mathrm{BM}}^{q}\left(x_{B}, k_{\perp}\right)=\lambda_{q} \Delta f_{\mathrm{Siv}}^{q}\left(x_{B}, k_{\perp}\right) .
$$

A legitimate question arises as to the compatibility of the two approaches i.e. whether Eqs. (60) and (27) are compatible. Here we study this question.

Under the assumption of Eqs. (60) one obtains:

$$
4 \Delta f_{\mathrm{BM}}^{Q_{V}}=\left(\lambda_{u}+\lambda_{d}+\lambda_{\bar{u}}+\lambda_{\bar{d}}\right) \Delta f_{\mathrm{Siv}}^{Q_{V}}+\Delta
$$

where

$$
\begin{aligned}
\Delta= & \left(3 \lambda_{u}-\lambda_{d}-\lambda_{\bar{u}}-\lambda_{\bar{d}}\right) \Delta f_{\mathrm{Siv}}^{u}+\left(\lambda_{u}+\lambda_{d}-3 \lambda_{\bar{u}}+\lambda_{\bar{d}}\right) \Delta f_{\mathrm{Siv}}^{\bar{u}} \\
& +\left(-\lambda_{u}+3 \lambda_{d}-\lambda_{\bar{u}}-\lambda_{\bar{d}}\right) f_{\mathrm{Siv}}^{d}+\left(\lambda_{u}+\lambda_{d}+\lambda_{\bar{u}}-3 \lambda_{\bar{d}}\right) \Delta f_{\mathrm{Siv}}^{\bar{d}} .
\end{aligned}
$$

Equation (61) is compatible with our assumption of proportionality Eq. (27) if

$$
|\Delta| \ll\left|\left(\lambda_{u}+\lambda_{d}+\lambda_{\bar{u}}+\lambda_{\bar{d}}\right) \Delta f_{\text {Siv }}^{Q_{V}}\right| \cdot
$$

Note that, at

$$
\lambda_{u}=\lambda_{d}=\lambda_{\bar{u}}=\lambda_{\bar{d}} \equiv \lambda_{Q_{V}},
$$

we have $\Delta=0$ and we obtain Eq. (27).

The values for $\lambda_{u, d}$ are those obtained in [4] assuming $\lambda_{\bar{u}}=-1, \lambda_{\bar{d}}=+1$ for the antiquarks i.e.

$$
\lambda_{u}=2.1 \pm 0.1, \quad \lambda_{d}=-1.111 \pm 0.001
$$

The parametrization of the Sivers function for each quark flavor is taken from [28]:

$$
\Delta f_{\mathrm{Siv}}^{q}\left(x_{B}, k_{\perp}\right)=\Delta f_{\mathrm{Siv}}^{q}\left(x_{B}\right) \sqrt{2 e} \frac{k_{\perp}}{M_{1}} \frac{e^{-k_{\perp}^{2} /\left\langle k_{\perp}^{2}\right\rangle_{S}}}{\pi\left\langle k_{\perp}^{2}\right\rangle_{S}}
$$

with

$$
\Delta f_{\text {Siv }}^{q}\left(x_{B}\right)=2 \mathcal{N}_{S}^{q}\left(x_{B}\right) q\left(x_{B}\right)
$$




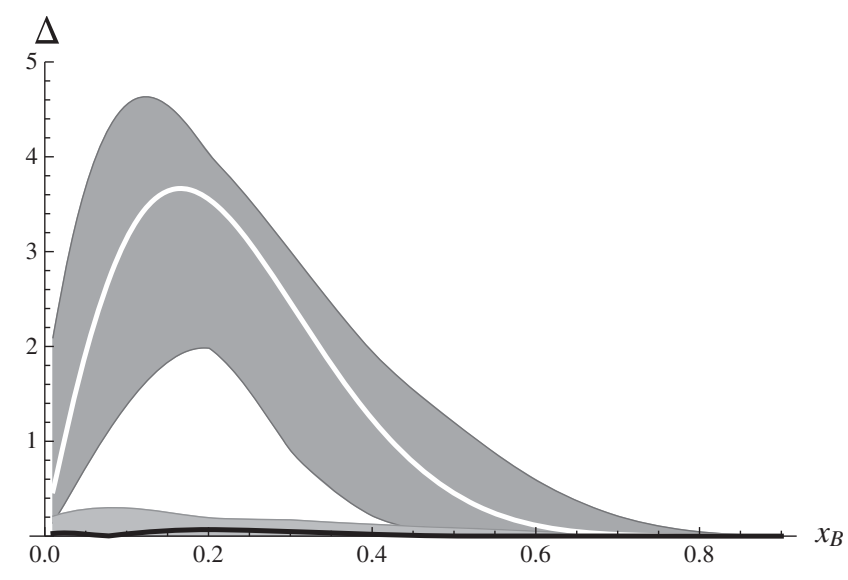

FIG. 6. A comparison between $\left|\left(\lambda_{u}+\lambda_{d}+\lambda_{\bar{u}}+\lambda_{\bar{d}}\right) \Delta f_{S}^{Q_{V}}\right|$ (the black curve which is almost 0 ) and $|\Delta|$ (white curve). The shaded areas are the corresponding statistical errors. The parametrization of $f_{\text {Siv }}^{q}$ is taken from [28] and the values of $\lambda_{q}$ are from [4].

$$
\mathcal{N}_{q}(x)=N_{q} x^{\alpha_{q}}(1-x)^{\beta_{q}} \frac{\left(\alpha_{q}+\beta_{q}\right)^{\alpha_{q}+\beta_{q}}}{\alpha_{q}^{\alpha_{q}} \beta_{q}^{\beta_{q}}}
$$

where:

$$
\begin{aligned}
N_{u} & =0.35 \pm 0.08, \quad N_{d}=-0.90_{-0.10}^{+0.43} \\
N_{\bar{u}} & =0.04_{-0.24}^{+0.22}, \quad N_{\bar{d}}=-0.40_{-0.44}^{+0.33} \\
\alpha_{u} & =0.73_{-0.58}^{+0.72}, \quad \alpha_{d}=1.08_{-0.65}^{+0.82}, \quad \alpha_{\text {sea }}=0.79_{-0.47}^{+0.56} \\
\beta_{q} \equiv \beta & =3.46_{-2.90}^{+4.87}, \quad M_{1}^{2}=0.34_{-0.16}^{+0.30}(\mathrm{GeV} / c)^{2}
\end{aligned}
$$

As the dependence on $k_{\perp}^{2}$ is the same for both the BM and Sivers functions, in Fig. 6 we compare only the dependence on $x_{B}$ of the two functions $\left(\lambda_{u}+\lambda_{d}+\lambda_{\bar{u}}+\lambda_{\bar{d}}\right) \Delta f_{\text {Siv }}^{Q_{V}}\left(x_{B}\right)$ and $\Delta\left(x_{B}\right)$. For the unpolarized PDFs the CTEQ6 parametrization was used.

From this figure it is clear that, even accounting for the enormous errors induced by the errors of the Sivers functions, $|\Delta|$ is much bigger than $\left|\left(\lambda_{u}+\lambda_{d}+\lambda_{\bar{u}}+\lambda_{\bar{d}}\right) \Delta f_{\text {Siv }}^{Q_{V}}\right|$, which is just the opposite to Eq. (63). This suggests that the extraction of the Boer-Mulders function in the literature $[3,4]$ is unreliable.

\section{CONCLUSIONS}

We had shown previously [2] that data on difference asymmetries allow one to test the assumed relation of proportionality between the BM and Sivers functions, which is currently used in the extraction of the BM function from data. In the present paper we perform two independent tests of this assumption applied, however, to the sum of the valence-quark TMD distributions, (27), using the COMPASS SIDIS data $[20,21]$ on the difference asymmetries $A_{U U, d}^{\cos \phi_{h}, h^{+}-h^{-}}\left(x_{B}\right), A_{U U, d}^{\cos 2 \phi_{h}, h^{+}-h^{-}}\left(x_{B}\right)$ and $A_{U T, d}^{\operatorname{Siv}, h^{+}-h^{-}}\left(x_{B}\right)$. Both tests are consistent with this assumption in the same kinematic interval $x_{B}=[0.014,0.13]$.
However, in the published extractions of the BM functions $[3,4]$, obtained in a completely different kind of analysis, based on the available parametrizations of both Sivers and Collins functions, it is assumed that BM and Sivers functions are proportional for each quark and antiquark separately [Eq. (60)]. This would agree with our result, based only on measurable quantities, if $\lambda_{u} \approx \lambda_{\bar{u}} \approx \lambda_{d} \approx \lambda_{\bar{d}} \approx \lambda_{Q_{V}}$, which does not correspond to the values and their errors obtained in $[3,4]$.

We have also determined the kinematical Cahn contribution, both directly from a fit to the data (as far as we know for the first time) and from a calculation. The calculated values are very sensitive to the average transverse momentum-squared, $\left\langle k_{\perp}^{2}\right\rangle$ and $\left\langle p_{\perp}^{2}\right\rangle$ in the unpolarized PDFs and FFs, respectively. Surprisingly, both for $A_{U U, d}^{\cos \phi_{h}, h^{+}-h^{-}}\left(x_{B}\right)$ and $A_{U U, d}^{\cos 2 \phi_{h}, h^{+}-h^{-}}\left(x_{B}\right)$, the calculated values agree with the extracted ones only for average transverse momenta close to the old experimental values, $\left\langle k_{\perp}^{2}\right\rangle=0.18$ and $\left\langle k_{\perp}^{2}\right\rangle=0.25,\left\langle p_{\perp}^{2}\right\rangle=0.20 \mathrm{GeV}^{2}$ and completely disagree with the much bigger present-day estimates. On smaller values for the intrinsic transverse momenta was suggested also in the covariant parton model $[29,30]$.

\section{ACKNOWLEDGMENTS}

We are grateful to Fabienne Kunne and Anna Martin for helpful comments concerning the COMPASS data, and E. C. acknowledges helpful discussions on the Cahn effect. E. C. and M. S. acknowledge the support of Grant No. 08-17/ 2016 of the Bulgarian Science Foundation. E. L. is grateful to the Leverhulme Trust for an Emeritus Fellowship.

\section{APPENDIX A: THE $A_{U U}^{\cos \phi_{h}}$ ASYMMETRY}

The structure function $F_{U U}^{\cos \phi_{h}}$ that determines the azimuthal $A_{U U}^{\cos \phi_{h}}$ asymmetry, Eq. (24), has two twist-3 contributions of $1 / Q$-order from the Cahn effect and the BM TMDs:

$$
F_{U U}^{\cos \phi_{h}}=F_{\mathrm{Cahn}}^{\cos \phi_{h}}+F_{\mathrm{BM}}^{\cos \phi_{h}} .
$$

For the difference cross sections $(h-\bar{h})$ on the deuteron target, it is only the sum of the valence-quark parton densities $Q_{V}$ enter these functions and for $h=\pi^{+}, K^{+}, h^{+}$ they read [8]

$$
\begin{aligned}
F_{\mathrm{Cahn}, d}^{\cos \phi_{h}, h-\bar{h}}= & \frac{2}{Q} Q_{V}\left(x_{B}, Q^{2}\right)\left[D_{q_{V}}^{h}\left(z_{h}, Q^{2}\right)\right] \mathcal{A}_{\mathrm{Cahn}}^{\cos \phi_{h}}\left(z_{h}, P_{T}^{2}\right) \\
F_{\mathrm{BM}, d}^{\cos \phi_{h}, h-\bar{h}}= & \frac{2}{Q} \Delta f_{\mathrm{BM}}^{Q_{V}}\left(x_{B}, Q^{2}\right)\left[\Delta^{N} D_{q_{V} \uparrow}^{h}\left(z_{h}, Q^{2}\right)\right] \\
& \times \mathcal{A}_{\mathrm{BM}}^{\cos \phi_{h}}\left(z_{h}, P_{T}^{2}\right)
\end{aligned}
$$


The functions $\mathcal{A}_{\mathrm{Cahn}}^{\cos \phi_{h}}$ and $\mathcal{A}_{\mathrm{BM}}^{\cos \phi_{h}}$ are independent of quark flavor and of the final hadron $h$ :

$$
\begin{gathered}
\mathcal{A}_{\mathrm{Cahn}}^{\cos \phi_{h}}\left(z_{h}, P_{T}^{2}\right)=-P_{T} z_{h}\left\langle k_{\perp}^{2}\right\rangle \frac{e^{-P_{T}^{2} /\left\langle P_{T}^{2}\right\rangle}}{\pi\left\langle P_{T}^{2}\right\rangle^{2}} \\
\mathcal{A}_{\mathrm{BM}}^{\cos \phi_{h}}\left(z_{h}, P_{T}^{2}\right)=e K P_{T} \frac{e^{-P_{T}^{2} /\left\langle P_{T}^{2}\right\rangle_{\mathrm{BM}}}}{\pi\left\langle P_{T}^{2}\right\rangle_{\mathrm{BM}}^{4}}\left[\left(-z_{h}^{2}\left\langle k_{\perp}^{2}\right\rangle_{\mathrm{BM}}+\left\langle p_{\perp}^{2}\right\rangle_{C}\right)\left\langle P_{T}^{2}\right\rangle_{\mathrm{BM}}+z_{h}^{2}\left\langle k_{\perp}^{2}\right\rangle_{\mathrm{BM}} P_{T}^{2}\right] \\
K \equiv \frac{\left\langle k_{\perp}^{2}\right\rangle_{\mathrm{BM}}^{2}\left\langle p_{\perp}^{2}\right\rangle_{C}^{2}}{\left\langle k_{\perp}^{2}\right\rangle\left\langle p_{\perp}^{2}\right\rangle} .
\end{gathered}
$$

Here the notation $\left[D_{q_{V}}^{h}\right]$ and $\left[\Delta^{N} D_{q_{V} \uparrow}^{h}\left(z_{h}\right)\right]$ stand for combinations of the valence-quark collinear and TMD FFs defined in Eqs. (35)-(38).

We can perform the integration over $P_{T}$ analytically, and we obtain

$$
\int d P_{T}^{2} F_{U U, d}^{\cos \phi_{h}, h-\bar{h}}=\frac{2}{\sqrt{\pi} Q} Q_{V}\left(x_{B}, Q^{2}\right)\left\{A_{\mathrm{Cahn}}^{h}\left(z_{h} \cdot Q^{2}\right)+2 \mathcal{N}_{\mathrm{BM}}\left(x_{B}\right) A_{\mathrm{BM}}^{h}\left(z_{h}, Q^{2}\right)\right\},
$$

where we have used the standard parametrization Eq. (11) for the BM function, and the notation $A_{\mathrm{Cahn,BM}}^{h}$ stands for

$$
\begin{gathered}
A_{\mathrm{Cahn}}^{h}\left(z_{h} \cdot Q^{2}\right) \equiv \sqrt{\pi}\left[D_{u_{V}}^{h}\left(z_{h}, Q^{2}\right)\right] \int d P_{T}^{2} \mathcal{A}_{\mathrm{Cahn}}^{\cos \phi_{h}}\left(z_{h}, P_{T}^{2}\right)=-\frac{z_{h}\left\langle k_{\perp}^{2}\right\rangle}{2 \sqrt{\left\langle P_{T}^{2}\right\rangle}}\left[D_{u_{V}}^{h}\left(z_{h}, Q^{2}\right)\right] \\
A_{\mathrm{BM}}^{h}\left(z_{h} \cdot Q^{2}\right) \equiv \sqrt{\pi}\left[\Delta^{N} D_{u_{V} \uparrow}^{h}\left(z_{h}, Q^{2}\right)\right] \int d P_{T}^{2} \mathcal{A}_{\mathrm{BM}}^{\cos \phi_{h}}\left(z_{h}, P_{T}^{2}\right) \\
=\frac{e K}{4 M_{\mathrm{BM}} M_{C}\left\langle P_{T}^{2}\right\rangle_{\mathrm{BM}}^{3 / 2}}\left[z_{h}^{2}\left\langle k_{\perp}^{2}\right\rangle_{\mathrm{BM}}+2\left\langle p_{\perp}^{2}\right\rangle_{C}\right]\left[\Delta^{N} D_{u_{V} \uparrow}^{h}\left(z_{h}, Q^{2}\right)\right] .
\end{gathered}
$$

For the unpolarized function $F_{U U, d}^{h-\bar{h}}$, which normalizes the asymmetry, we have

$$
\int d P_{T}^{2} F_{U U, d}^{h-\bar{h}}=\frac{1}{\pi} Q_{V}\left(x_{B}, Q^{2}\right)\left[D_{u_{V}}^{h}\left(z_{h}, Q^{2}\right)\right]
$$

Thus, for the integrated over $P_{T}^{2}$ asymmetry $A_{U U, d}^{\cos \phi_{h}, h-\bar{h}}$, we obtain

$$
A_{U U, d}^{\cos \phi_{h}, h-\bar{h}}\left(x_{B}, z_{h}, Q^{2}\right)=\frac{2 \sqrt{\pi}\left[(2-y) \sqrt{1-y} / Q^{5}\right] Q_{V}\left(x_{B}, Q^{2}\right)\left[A_{\mathrm{Cahn}}^{h}\left(z_{h} \cdot Q^{2}\right)+2 \mathcal{N}_{\mathrm{BM}}\left(x_{B}\right) A_{\mathrm{BM}}^{h}\left(z_{h}, Q^{2}\right)\right]}{\left[\left[1+(1-y)^{2}\right] / Q^{4}\right] Q_{V}\left(x_{B}, Q^{2}\right)\left[D_{u_{V}}^{h}\left(z_{h}, Q^{2}\right)\right]} .
$$

From this expression, it follows that if one can neglect $Q^{2}$ dependence in $Q_{V}\left(x_{B}, Q^{2}\right)$ and in the FFs, the $x_{B}$ and $z_{h}$ dependencies will factorize. Also, $Q_{V}\left(x_{B}\right)$ in the numerator and denominator cancel out and for the $x_{B}$-dependent difference asymmetry on the deuteron $A_{U U, d}^{\cos \phi_{h}, h-\bar{h}}\left(x_{B}\right)$, we obtain

$$
A_{U U, d}^{\cos \phi_{h}, h-\bar{h}}\left(x_{B}\right)=\frac{2 \sqrt{\pi} \int d Q^{2}\left[(2-y) \sqrt{1-y} / Q^{5}\right] \int d z_{h}\left[A_{\mathrm{Cahn}}^{h}\left(z_{h}, Q^{2}\right)+2 \mathcal{N}_{\mathrm{BM}}\left(x_{B}\right) A_{\mathrm{BM}}^{h}\left(z_{h}, Q^{2}\right)\right]}{\left.\int d Q^{2}\left[1+(1-y)^{2}\right] / Q^{4}\right] \int d z_{h}\left[D_{u_{V}}^{h}\left(z_{h}, Q^{2}\right)\right]} .
$$

Further, after neglecting $Q^{2}$ dependence in the collinear FFs, and replacing the integration over $Q^{2}$ by $\Delta Q^{2}$ times the function evaluated at some average value $\langle Q\rangle$ (or equivalently $\bar{y}$ ) for each $x_{B}$ bin, we obtain the simple $x_{B}$-dependent expression for the asymmetry:

$$
A_{U U, d}^{\cos \phi_{h}, h-\bar{h}}\left(x_{B}\right)=\Phi\left(x_{B}\right)\left\{C_{\text {Cahn }}^{h}+2 \mathcal{N}_{\mathrm{BM}}^{Q_{V}}\left(x_{B}\right) C_{\mathrm{BM}}^{h}\right\}, \quad h=\pi^{+}, K^{+}, h^{+} .
$$

The function $\Phi\left(x_{B}\right)$ is given in Eq. (33), it is completely fixed by kinematics, the same for all final hadrons. The constants $C_{\mathrm{Cahn}}^{h}$ and $C_{\mathrm{BM}}^{h}$ are determined by the expressions 


$$
\begin{gathered}
C_{\mathrm{Cahn}}^{h}=\frac{2 \int d z_{h} A_{\mathrm{Cahn}}^{h}\left(z_{h}\right)}{\int d z_{h}\left[D_{u_{V}}^{h}\left(z_{h}\right]\right.}=-\left\langle k_{\perp}^{2}\right\rangle \frac{\int d z_{h} z_{h}\left[D_{q_{V}}^{h}\left(z_{h}\right)\right] / \sqrt{\left\langle P_{T}^{2}\right\rangle}}{\int d z_{h}\left[D_{q_{V}}^{h}\left(z_{h}\right)\right]}, \\
C_{\mathrm{BM}}^{h}=\frac{2 \int d z_{h} A_{\mathrm{BM}}^{h}\left(z_{h}\right)}{\int d z_{h}\left[D_{u_{V}}^{h}\left(z_{h}\right]\right.} \\
=\frac{e\left\langle k_{\perp}^{2}\right\rangle_{\mathrm{BM}}^{2}\left\langle p_{\perp}^{2}\right\rangle_{C}^{2}}{2 M_{\mathrm{BM}} M_{C}\left\langle k_{\perp}^{2}\right\rangle\left\langle p_{\perp}^{2}\right\rangle} \frac{\int d z_{h}\left[z_{h}^{2}\left\langle k_{\perp}^{2}\right\rangle_{\mathrm{BM}}+2\left\langle p_{\perp}^{2}\right\rangle_{C}\right]\left[\Delta^{N} D_{q_{V} \uparrow}^{h}\left(z_{h}\right)\right] /\left\langle P_{T}^{2}\right\rangle_{\mathrm{BM}}^{3 / 2}}{\int d z_{h}\left[D_{u_{V}}^{h}\left(z_{h}\right)\right]} \\
\left\langle P_{T}^{2}\right\rangle=\left\langle p_{\perp}^{2}\right\rangle+z_{h}^{2}\left\langle k_{\perp}^{2}\right\rangle, \quad\left\langle P_{T}^{2}\right\rangle_{\mathrm{BM}}=\left\langle p_{\perp}^{2}\right\rangle_{C}+z_{h}^{2}\left\langle k_{\perp}^{2}\right\rangle_{\mathrm{BM}} .
\end{gathered}
$$

\section{APPENDIX B: THE $A_{U U}^{\cos 2 \phi_{h}}$ ASYMMETRY}

The structure function $F_{U U}^{\cos 2 \phi_{h}}$ that determines the azimuthal $A_{U U}^{\cos 2 \phi_{h}}$ asymmetry, Eq. (25), has two contributions-the leading twist- 2 contribution from the BM functions and the twist- 4 contribution of $1 / Q^{2}$ order from the Cahn effect:

$$
F_{U U}^{\cos 2 \phi_{h}}=F_{\mathrm{Cahn}}^{\cos 2 \phi_{h}}+F_{\mathrm{BM}}^{\cos 2 \phi_{h}} .
$$

Again we shall consider only difference cross sections $(h-\bar{h})$ on the deuteron target. In this case, it is only the sum of the valence-quark parton densities $Q_{V}$ that enter these functions.

For the BM contribution on the deuteron target for $h=\pi^{+}, K^{+}, h^{+}$, we have [8]

$$
F_{\mathrm{BM}, d}^{\cos 2 \phi_{h}, h-\bar{h}}\left(x_{B}, z_{h}, Q^{2}, P_{T}^{2}\right)=\mathcal{A}_{\mathrm{BM}}^{\cos 2 \phi_{h}}\left(z_{h}, P_{T}^{2}\right) \Delta f_{\mathrm{BM}}^{Q_{V}}\left(x_{B}, Q^{2}\right)\left[\Delta^{N} D_{q_{V} \uparrow}^{h}\left(z_{h}, Q^{2}\right)\right],
$$

where the flavor- and hadron-independent function $\mathcal{A}_{\mathrm{BM}}^{\cos 2 \phi_{h}}\left(z_{h}, P_{T}^{2}\right)$ reads

$$
\mathcal{A}_{\mathrm{BM}}^{\cos 2 \phi_{h}}\left(z_{h}, P_{T}^{2}\right)=-e K \frac{P_{T}^{2}}{M_{\mathrm{BM}} M_{C}} \frac{e^{-P_{T}^{2} /\left\langle P_{T}^{2}\right\rangle_{\mathrm{BM}}}}{\pi\left\langle P_{T}^{2}\right\rangle_{\mathrm{BM}}^{3}} z_{h},
$$

and $K$ is determined in Eq. (A5).

Performing the integration over $P_{T}$ and using the standard parametrization Eq. (11) for the BM function, we obtain

$$
\int d P_{T}^{2} F_{U U, d}^{\cos 2 \phi_{h}, h-\bar{h}}=2 \hat{A}_{\mathrm{BM}}^{h}\left(z_{h}, Q^{2}\right) \mathcal{N}_{\mathrm{BM}}^{Q_{V}}\left(x_{B}\right) Q_{V}\left(x_{B}, Q^{2}\right),
$$

where

$$
\hat{A}_{\mathrm{BM}}^{h}\left(z_{h}, Q^{2}\right)=\left[\Delta^{N} D_{u_{V} \uparrow}^{h}\left(z_{h}, Q^{2}\right)\right] \int d P_{T}^{2} \mathcal{A}_{\mathrm{BM}}^{\cos 2 \phi_{h}}\left(z_{h}, P_{T}^{2}\right)=\frac{-e K z_{h}}{\pi M_{\mathrm{BM}} M_{C}\left\langle P_{T}^{2}\right\rangle_{\mathrm{BM}}}\left[\Delta^{N} D_{u_{V} \uparrow}^{h}\left(z_{h}, Q^{2}\right)\right] .
$$

Equation (B4) implies that if we can neglect $Q^{2}$ dependencies in $Q_{V}$ and in the FF, the $x_{B}$ and $z_{h}$ dependencies will factorize, and $\left[\Delta^{N} D_{u_{V} \uparrow}^{h}\right]$ is given in Eq. (38).

The Cahn contribution to the asymmetry looks more complicated as the integration over $k_{\perp}$ that comes from the convolution of the TMD PDFs and FFs cannot be fulfilled analytically. Nevertheless, it has the same structure:

$$
\begin{aligned}
F_{\mathrm{Cahn}, d}^{\cos 2 \phi_{h}, h-\bar{h}}\left(x_{B}, z_{h}, Q^{2}, \mathbf{P}_{T}\right)= & \frac{2}{Q^{2}} Q_{V}\left(x_{B}, Q^{2}\right)\left[D_{q_{V}}^{h}\left(z_{h}, Q^{2}\right)\right] \\
& \times \int d^{2} \mathbf{k}_{\perp} d^{2} \mathbf{p}_{\perp}\left(2\left(\hat{\mathbf{P}_{\mathbf{T}}} \cdot \mathbf{k}_{\perp}\right)^{2}-k_{\perp}^{2}\right) \frac{e^{-k_{\perp}^{2} /\left\langle k_{\perp}^{2}\right\rangle}}{\pi\left\langle k_{\perp}^{2}\right\rangle} \frac{e^{-k_{\perp}^{2} /\left\langle k_{\perp}^{2}\right\rangle}}{\pi\left\langle k_{\perp}^{2}\right\rangle} \delta^{2}\left(\mathbf{P}_{\mathbf{T}}-z_{h} \mathbf{k}_{\perp}-\mathbf{p}_{\perp}\right) \\
= & \frac{2}{Q^{2}} Q_{V}\left(x_{B}, Q^{2}\right)\left[D_{q_{V}}^{h}\left(z_{h}, Q^{2}\right)\right] \frac{1}{2 \pi^{2}\left\langle k_{\perp}^{2}\right\rangle\left\langle p_{\perp}^{2}\right\rangle} I\left(z_{h}, P_{T}^{2}\right),
\end{aligned}
$$

where $\left[D_{q_{V}}^{h}\right]$ is given in Eq. (35), and 


$$
I=e^{-\frac{P_{T}^{2}}{\left\langle p_{\perp}^{2}\right\rangle}} \int d k_{\perp}^{2} k_{\perp}^{2} e^{-k_{\perp}^{2} \frac{\left\langle P_{T}^{2}\right\rangle}{\left.\left\langle k_{\perp}^{2}\right\rangle p_{\perp}^{2}\right\rangle}} \int_{0}^{2 \pi} d \phi \cos 2 \phi e^{a \cos \phi}, \quad a=\frac{2 z_{h} k_{\perp} P_{T}}{\left\langle p_{\perp}^{2}\right\rangle} .
$$

For the integrated over $\mathbf{P}_{T}$ contribution of the Cahn effect, we obtain

$$
\int d^{2} \mathbf{P}_{T} F_{\mathrm{Cahn}, d}^{\cos 2 \phi_{h}, h-\bar{h}}=\frac{2}{Q^{2}} \hat{A}_{\mathrm{Cahn}}^{h}\left(z_{h}, Q^{2}\right) Q_{V}\left(x_{B}, Q^{2}\right),
$$

where

$$
\hat{A}_{\text {Cahn }}^{h}\left(z_{h}, Q^{2}\right) \equiv \frac{1}{2 \pi\left\langle k_{\perp}^{2}\right\rangle\left\langle p_{\perp}^{2}\right\rangle} J\left(z_{h}\right)\left[D_{q_{V}}^{h}\left(z_{h}, Q^{2}\right)\right], \quad J\left(z_{h}\right) \equiv \int d P_{T}^{2} I\left(z_{h}, P_{T}^{2}\right) .
$$

From Eqs. (B4) and (B8), and using Eq. (A9), we obtain the following expression for the asymmetry $A_{U U, d}^{\cos 2 \phi_{h}, h-\bar{h}}$ :

$$
A_{U U, d}^{\cos 2 \phi_{h}, h-\bar{h}}\left(x_{B}, z_{h}, Q^{2}\right)=\frac{2\left[(1-y) / Q^{4}\right] Q_{V}\left(x_{B}, Q^{2}\right)\left\{\hat{A}_{\mathrm{BM}}^{h}\left(z_{h}, Q^{2}\right) \mathcal{N}_{\mathrm{BM}}^{Q_{V}}\left(x_{B}\right)+\left[1 / Q^{2}\right] \hat{A}_{\mathrm{Cahn}}^{h}\left(z_{h}, Q^{2}\right)\right\}}{\left[\left[1+(1-y)^{2}\right] / Q^{4}\right] Q_{V}\left(x_{B}, Q^{2}\right)\left[D_{u_{V}}^{h}\left(z_{h}, Q^{2}\right)\right]} .
$$

Neglecting $Q^{2}$ dependence in the $x_{B}$ bins in $Q_{V}$, the valence quark densities $Q_{V}$ in the nominator and in the denominator cancel out. Neglecting further, the $Q^{2}$ dependence in the FFs and, integrating over $z_{h}$ for the $x_{B}$-dependent $\cos 2 \phi_{h}$ asymmetry, we obtain

$$
A_{U U, d}^{\cos 2 \phi_{h}, h-\bar{h}}\left(x_{B}\right)=\hat{\Phi}\left(x_{B}\right)\left\{\hat{C}_{\mathrm{BM}}^{h} \mathcal{N}_{\mathrm{BM}}^{Q_{V}}\left(x_{B}\right)+\frac{M M_{d}}{Q^{2}} \hat{C}_{\mathrm{Cahn}}^{h}\right\}
$$

where

$$
\begin{aligned}
\hat{\Phi}\left(x_{B}\right) & =\frac{2(1-\bar{y})}{\left[1+(1-\bar{y})^{2}\right]} \\
\hat{C}_{\mathrm{BM}}^{h} & =\frac{-e K}{M_{\mathrm{BM}} M_{C}} \frac{\int d z_{h} z_{h}\left[\Delta^{N} D_{u_{V} \uparrow}^{h}\left(z_{h}\right)\right] /\left\langle P_{T}^{2}\right\rangle_{\mathrm{BM}}}{\int d z_{h}\left[D_{u_{V}}^{h}\left(z_{h}\right)\right]} \\
\hat{C}_{\mathrm{Cahn}}^{h} & =\frac{1}{2 M M_{d}\left\langle k_{\perp}^{2}\right\rangle\left\langle p_{\perp}^{2}\right\rangle} \frac{\int d z_{h}\left[D_{u_{V}}^{h}\left(z_{h}\right)\right] \int d P_{T}^{2} I\left(z_{h}, P_{T}^{2}\right)}{\int d z_{h}\left[D_{u_{V}}^{h}\left(z_{h}\right)\right]},
\end{aligned}
$$

where $\bar{y}$ is given in Eq. (34). Equation (B11) is exactly our Eq. (45).

[1] M. Anselmino, M. Boglione, U. D’Alesio, S. Melis, F. Murgia, E. R. Nocera, and A. Prokudin, Phys. Rev. D 83, 114019 (2011).

[2] E. Christova and E. Leader, Phys. Rev. D 92, 114004 (2015).

[3] V. Barone, A. Prokudin, and B.-Q. Ma, Phys. Rev. D 78, 045022 (2008).

[4] V. Barone, S. Melis, and A. Prokudin, Phys. Rev. D 81, 114026 (2010).

[5] A. Bacchetta, F. Conti, and M. Radici, Phys. Rev. D 78, 074010 (2008); A. Courtoy, S. Scopetta, and V. Vento, Phys. Rev. D 80, 074032 (2009); B. Pasquini and F.Yuan, arXiv: 1001.5398
[6] M. Alekseev et al. (COMPASS Collaboration), Phys. Lett. B 660, 458 (2008).

[7] M. Anselmino, M. Boglione, J. O. Gonzalez H., S. Melis, and A. Prokudin, J. High Energy Phys. 04 (2014) 005.

[8] E. Christova, Phys. Rev. D 90, 054005 (2014).

[9] M. Anselmino, M. Boglione, U. D’Alesio, A. Kotzinian, F. Murgia, and A. Prokudin, Phys. Rev. D 71, 074006 (2005).

[10] M. Arneodo et al. (EMC Collaboration), Z. Phys. C 34, 277 (1987).

[11] M. R. Adams et al. (Fermilab E665 Collaboration), Phys. Rev. D 48, 5057 (1993).

[12] F. Giordano, Report No. DESY-THESIS-2008-030. 
[13] A. Airapetian et al. (HERMES Collaboration), Phys. Rev. D 87, 074029 (2013).

[14] C. Adolph et al. (COMPASS Collaboration), Eur. Phys. J. C 73, 2531 (2013).

[15] D. Sivers, Phys. Rev. D 41, 83 (1990); 43, 261 (1991).

[16] D. Boer and P. J. Mulders, Phys. Rev. D 57, 5780 (1998).

[17] J. C. Collins, Nucl. Phys. B396, 161 (1993).

[18] M. Anselmino, M. Boglione, U. D’Alesio, A. Kotzinian, F. Murgia, A. Prokudin, and C. Türk, Phys. Rev. D 75, 054032 (2007).

[19] M. Anselmino, M. Boglione, U. D’Alesio, S. Melis, F. Murgia, and A. Prokudin, Phys. Rev. D 87, 094019 (2013).

[20] C. Adolph et al. (COMPASS Collaboration), Nucl. Phys. B886, 1046 (2014).

[21] E. S. Ageev et al. (COMPASS Collaboration), Nucl. Phys. B765, 31 (2007); A. Martin, Czech. J. Phys. 56, F33 (2006).
[22] The Durham HepData Project, http://hepdata.cedar.ac.uk/ pdf/pdf3.html.

[23] E. Leader, A. V. Sidorov, and D. Stamenov, in Proceedings of the 15th Workshop on High Energy Physic (DSPIN-13), Dubna, Russia (2013), arXiv:1312.5200.

[24] A. Airapetain et al., Phys. Rev. D 87, 074029 (2013).

[25] N. Makke (for COMPASS Collaboration), Proc. Sci. DIS2013 (2013) 202.

[26] COMPASS Collaboration, arXiv:1604.02695

[27] S. Albino, B. A. Kniehl, and G. Kramer, Nucl. Phys. B803, 42 (2008).

[28] M. Anselmino, M. Boglione, U. D’Alesio, A. Kotzinian, S. Melis, F. Murgia, A. Prokudin, and C. Türk, Eur. Phys. J. A 39, 89 (2009).

[29] P. Zavada, Phys. Rev. D 83, 014022 (2011); 85, 037501 (2012).

[30] U. D’Alesio, E. Leader, and F. Murgia, Phys. Rev. D 81, 036010 (2010). 